\title{
Competition between intra-protein charge recombination and electron transfer outside photosystem I complexes used for photovoltaic applications
}

\author{
Alice Goyal ${ }^{1} \cdot$ Sebastian Szewczyk ${ }^{1}$. Gotard Burdziński ${ }^{1} \cdot$ Mateusz Abram $^{2,3} \cdot$ Joanna Kargull . \\ Krzysztof Gibasiewicz ${ }^{1}$ (1)
}

Received: 30 September 2021 / Accepted: 7 January 2022 / Published online: 4 February 2022

(c) The Author(s) 2022

\begin{abstract}
Photosystem I (PSI) complexes isolated from three different species were electrodeposited on FTO conducting glass, forming a photoactive multilayer of the photo-electrode, for investigation of intricate electron transfer (ET) properties in such green hybrid nanosystems. The internal quantum efficiency of photo-electrochemical cells (PEC) containing the PSI-based photo-electrodes did not exceed $\sim 0.5 \%$. To reveal the reason for such a low efficiency of photocurrent generation, the temporal evolution of the transient concentration of the photo-oxidized primary electron donor, $\mathrm{P}^{+}$, was studied in aqueous suspensions of the PSI complexes by time-resolved absorption spectroscopy. The results of these measurements provided the information on: (1) completeness of charge separation in PSI reaction centers (RCs), (2) dynamics of internal charge recombination, and (3) efficiency of electron transfer from PSI to the electrolyte, which is the reaction competing with the internal charge recombination in the PSI RC. The efficiency of the full charge separation in the PSI complexes used for functionalization of the electrodes was $\sim 90 \%$, indicating that incomplete charge separation was not the main reason for the small yield of photocurrents. For the PSI particles isolated from a green alga Chlamydomonas reinhardtii, the probability of ET outside PSI was 30-40\%, whereas for their counterparts isolated from a cyanobacterium Synechocystis sp. PCC 6803 and a red alga Cyanidioschyzon merolae, it represented a mere $\sim 4 \%$. We conclude from the transient absorption data for the PSI biocatalysts in solution that the observed small photocurrent efficiency of $\sim 0.5 \%$ for all the PECs analyzed in this study is likely due to: (1) limited efficiency of ET outside PSI, particularly in the case of PECs based on PSI from Synechocystis and C. merolae, and (2) the electrolyte-mediated electric short-circuiting in PSI particles forming the photoactive layer, particularly in the case of the $C$. reinhardtii PEC.
\end{abstract}

Graphical abstract

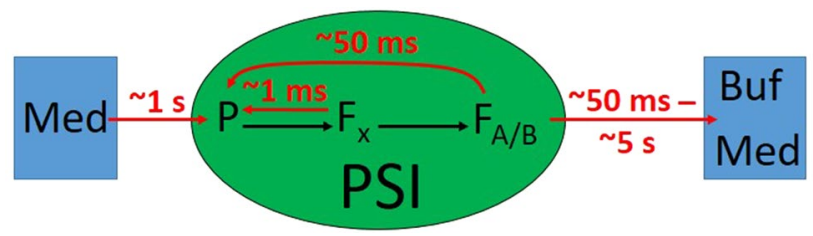

Keywords Photosystem I - Electron transfer · Charge recombination · Transient absorption · Photovoltaics · Photoelectrochemical cell

Krzysztof Gibasiewicz

krzyszgi@amu.edu.pl

Extended author information available on the last page of the article 


\section{Introduction}

Photosynthetic proteins, e.g., Photosystem I (PSI) and purple bacteria reaction centers, catalyzing light-induced charge separation are often used as the photoactive modules of semi-artificial solar cells and photo-electrochemical devices [1-6]. This is because these complexes show an exceptionally high efficiency of conversion of absorbed photons into a current of near $100 \%$ [7, 8]. However, the net efficiencies of such bio-hybrid devices, estimated on the basis of the amount of either incident or absorbed light and electric current generated in an external circuit, are usually much lower. Three types of efficiencies are usually reported for solar cell characterization: (1) external efficiency $\left(\eta=P_{\text {out }} / P_{\text {in }}\right)$ defined as a ratio of the power of electrical photocurrent $\left(P_{\text {out }}\right)$ and the power of incident light $\left(P_{\text {in }}\right),(2)$ incident photon to current efficiency (IPCE) or external quantum efficiency (EQE) defined as a ratio of the number of photo-generated electrons to the number of incident photons, and (3) internal quantum efficiency (IQE) defined as a ratio of the number of photo-generated electrons to the number of absorbed photons. For the PSI-based devices, the reported $\eta$ values never exceed $\sim 0.5 \%$ ([9]-0.001\%, [10]-0.08\%, [11]— $0.17 \%,[12]-0.52 \%)$, EQE does not exceed $11 \%$ ([12]—5\%, [13]- $0.01 \%,[14]-5 \%,[15]-11 \%$,), whereas IQE does not exceed 39\% ([14]-5\%, [15]—39\%, [16]-0.37\%). Only the last parameter can be directly compared to the $\sim 100 \%$ intrinsic "IQE" value characteristic of the photosynthetic proteins.

Often in the literature, only absolute photocurrent values are reported, and the respective conversion efficiencies are not [17-22]. There are a few possible reasons for the generally low performance of PSI-based devices [23]. They include disturbance of the native electron transfer (ET) inside the PSI complex incorporated into an artificial environment, a poor electronic connection between PSI particles and artificial components of the device, and back reactions (charge recombination and/or short-circuiting), all of which limit forward ET of the high-energy electrons generated upon absorption of solar energy within the PSI complex. On the other hand, relatively high efficiencies of some of the reported devices may result not from the native PSI performance, but rather from the specific experimental conditions that may affect the native structure and function of this complex, e.g., causing its chemical modification [10, 12]. Therefore, it is important to always correlate the efficiencies of the protein-based photovoltaic devices with the level of intactness of the photoactive proteins following their immobilization on the electrode surface.

The PSI bio-photocatalyst is a pigment-protein complex of oxygenic photosynthesis, whose core domain, largely structurally conserved between different species, comprises two large membrane-bound proteins ( $\mathrm{PsaA} / \mathrm{PsaB}$ heterodimer) which

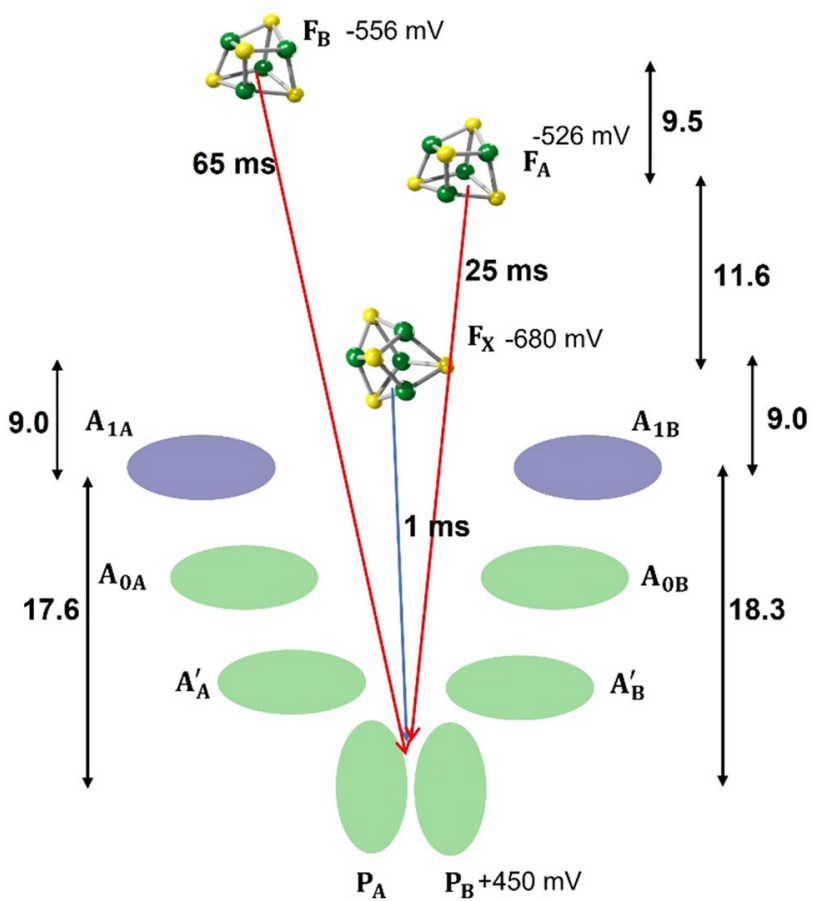

Fig. 1 Arrangement of PSI ET cofactors, redox midpoint potentials of iron-sulfur clusters $F_{X}, F_{A}, F_{B}$ [28], and time constants of back ET from iron-sulfur clusters to the primary electron donor [36]. The values at double black arrows represent edge-to-edge distances between individual cofactors (in $\AA$; [28]). Green ovals - Chls, purple ovals phylloquinones

provide a scaffold for binding the majority of the inner antenna system of 96 chlorophylls (Chls) and $22 \beta$-carotenes (Cars) as well as most of the electron transfer cofactors (ETC) [24-27]. The ETC system may be divided into two groups (Fig. 1). One of them, containing the initial ET carriers, is composed of two quasi-symmetric branches (denoted with subscripts A and B in the PSI X-ray structures [24-27]), each containing three Chls $a\left(P_{\mathrm{A} / \mathrm{B}}, A_{\mathrm{A} / \mathrm{B}}, A_{0 \mathrm{~A} / \mathrm{B}}\right)$ and one phylloquinone $\left(A_{1 \mathrm{~A} / \mathrm{B}}\right)$. The second group forms a single branch composed of three iron-sulfur [4Fe-4S] clusters $\left(F_{X}, F_{A}\right.$ and $\left.F_{B}\right)$, two of which, $F_{A}$ and $F_{B}$, are bound within the peripheral subunit PsaC, while $F_{X}$ is coordinated between the PsaA and PsaB subunits of the PSI core on the stromal side of the thylakoid membrane [24]. In a classical view [29-31], the ET starts at the primary electron donor $(P)$ which is a dimer of strongly interacting Chls $a\left(P_{A}\right.$ and $P_{B}$ ) positioned in the two branches. An electron is transferred from $P$ to $A_{0}$ and next to $A_{1}$ along both branches with varying relative efficiencies depending on the species [32-35]. Next, the electron is transferred from either of the two phylloquinones to $F_{X}$ and then to $F_{A}$ and $F_{B}$ clusters. Although $F_{B}$ is usually regarded as the terminal acceptor, it is not clear to which extent the electron is thermally equilibrated between $F_{A}$ and $F_{B}$. Therefore, the final charge separated state is often 


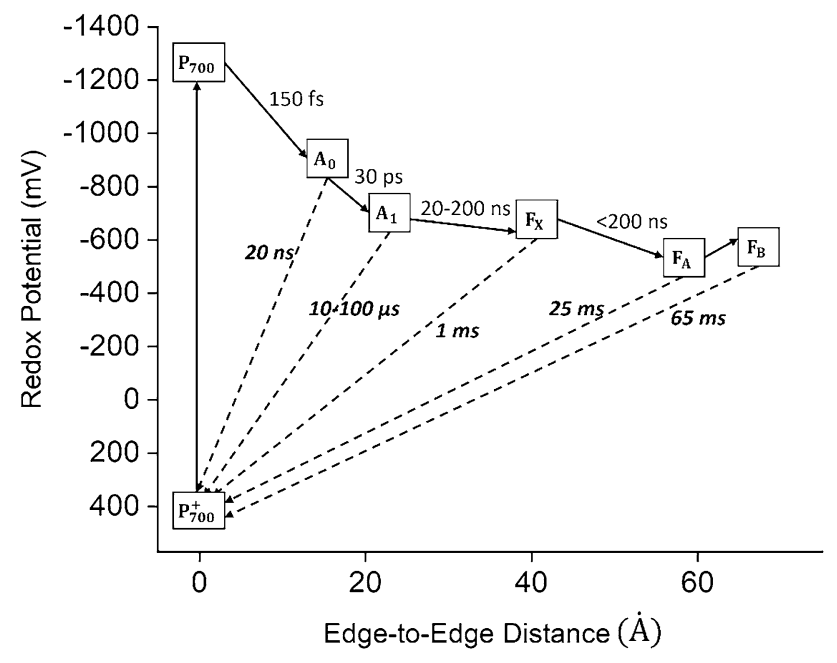

Fig. 2 Energetic-kinetic-distance scheme of cofactors in PSI (adapted from [36]). Solid arrows represent photoexcitation of $\mathrm{P}$ and forward ET reactions, whereas the dashed arrows represent back ET reactions

labeled as $P^{+}\left(F_{A} / F_{B}\right)^{-}$and the $\left(F_{A} / F_{B}\right)$ species is commonly treated as a single final acceptor $[29,36]$.

The forward ET inside intact PSI occurs on a time scale ranging from (sub)picoseconds to hundreds of nanoseconds and is practically not affected by back ET which occurs on a much slower time scale (see Fig. 2 and [29, 30, 36]). However, under conditions that disturb forward ET, back reactions are often observed on time scales ranging from nanoseconds to milliseconds (Figs. 1 and 2). Even in very good PSI preparations I, most of the different phases of internal charge recombination are observed. For example, Kurashov et al. [36] reported that $4 \%$ of the entire PSI population underwent microsecond (12-230 $\mu$ s) charge recombination between phylloquinones and the primary donor $\left(P^{+} A_{1 A / B}{ }^{-} \rightarrow P A_{1 A / B}\right)$, another $4 \%$ underwent charge recombination between $F_{X}$ cluster and $P$ within $\sim 2 \mathrm{~ms}$ $\left(P^{+} F_{X}{ }^{-} \rightarrow P F_{X}\right)$ and the major fraction of PSI complexes (56\%) underwent charge recombination between the terminal electron acceptor $\left(F_{A} / F_{B}\right)$ and the primary donor $P$ $\left(P^{+}\left(F_{A} / F_{B}\right)^{-} \rightarrow P\left(F_{A} / F_{B}\right)\right)$. The charge recombination was not observed in the remaining $36 \%$ of the PSI population, likely due to ET from $\left(F_{A} / F_{B}\right)^{-}$to one of the redox species present in the electrolyte (oxidized DCPIP or ascorbate). Such possibility is justified by the highly negative redox potential of $F_{A} / F_{A}{ }^{-}$and $F_{B} / F_{B}{ }^{-}$species $(\sim-550 \mathrm{mV}$; Fig. 1$)$, which makes them strongly reducing and thus, strongly reactive.

In this work, we investigated the transient absorption characteristics of five types of PSI complexes suspended in aqueous solutions in the presence or absence of external ET mediators and related those to the ET properties within the PSI-based devices. These PSI complexes, differing in their structures, show a low photovoltaic activity following electrodeposition on the conducting glass and the formation of a thin film. Our main aim was to examine to what extent the PSI complexes undergo complete charge separation in the reaction centers (RCs) and correlate the efficiency of this process with the observed photovoltaic output. Second, we examined whether upon complete charge separation, photo-generated electrons are efficiently transferred to the exogenous ET mediators, or whether charge recombination inside PSI RC competes with the external ET process (between PSI and electrolyte), which may significantly limit the efficiency of photocurrent generation.

\section{Materials and methods}

\subsection{Preparation of PSI particles}

PSI particles were isolated from three different species: a cyanobacterium Synechocystis sp. PCC 6803, and two eukaryotic phototrophs, i.e., a thermo-acidophilic red microalga Cyanidioschyzon merolae and a mesophilic green alga Chlamydomonas reinhardtii CC2696. Two forms of cyanobacterial PSI core complexes were studied-monomeric (SM) and trimeric (ST). They were prepared according to the procedure described in [37]. C. merolae PSI particles were isolated in two different structural forms obtained from cells grown in different growth conditions, as described elsewhere [38, 39]. PSI complexes grown under high light intensity $(\mathrm{CmH} ; 350$ $\mu \mathrm{E} \mathrm{m}^{-2} \mathrm{~s}^{-1}$ ) were composed of the PSI core and the peripheral antenna, light-harvesting complex I (LHCI), containing on average four Lhcr antenna subunits, while PSI particles obtained from the cells grown under medium light intensity ( $\mathrm{CmM}$; $90 \mu \mathrm{E} \mathrm{m}^{-2} \mathrm{~s}^{-1}$ ) were composed of the PSI core and LHCI containing on average six external Lhcr subunits. Thus, the smaller $\mathrm{CmH}$ particles contained 159 antenna Chls per $\mathrm{P}$, and larger CmM complexes - 186 antenna Chls per RC [38]. The procedure of isolation of PSI from C. reinhardtii (Cr) has been described elsewhere [33, 40]. The estimated number of Chls in the PSI core complexes obtained from this species ranges from 60 [41] to 85 Chls [42] although in the recent cryoEM studies of PSI-LHCI particles from a wild type $C$. reinhardtii strain, as many as 96 Chls were assigned to PSI core [43]. Complexes from all the species were extracted using $n$-dodecyl- $\beta$-D-maltoside ( $\beta$-DM) and purified by ion exchange chromatography [38, 39]. Isolated cyanobacterial PSI complexes were stored at $-20{ }^{\circ} \mathrm{C}$ without addition of glycerol, whereas the remaining PSI samples were kept frozen in the presence of $20-25 \%(w / v)$ glycerol at $-55{ }^{\circ} \mathrm{C}(C$. merolae $)$ or at $-20{ }^{\circ} \mathrm{C}(C$. reinhardtii $)$ prior to spectroscopic and electrochemical experiments.

\subsection{Immobilization of PSI on FTO conducting glass}

The immobilization procedure was described in detail in previous works [16, 37]. Briefly, $30 \mu \mathrm{l}$ of dialyzed PSI solution 
of optical density $\mathrm{OD}_{679 \mathrm{~nm}, 1 \mathrm{~cm}} \approx 1$ was drop-casted onto the conductive surface of the FTO slide and then covered with another FTO slide with its conductive layer facing the solution. The distance between the two slides was ensured by the application of a spacer of $\sim 1.5 \mathrm{~mm}$ in thickness. Next, a positive potential difference $(2.5 \mathrm{~V})$ was applied between the conductive surfaces of two electrodes for $5 \mathrm{~min}$. In such previously optimized conditions [16], a preferential migration of PSI particles toward the positive electrode could be observed. After that, the samples were air-dried overnight at $4{ }^{\circ} \mathrm{C}$ to evaporate the solvent. The approximated area of the resultant PSI layer was $\sim 0.25 \mathrm{~cm}^{2}$, and its absorption UV-vis spectrum was measured using a Hitachi U-2800A spectrophotometer. The absorbance of the dried immobilized PSI complexes at the $\mathrm{Q}_{\mathrm{y}}$ band maximum was measured as $A_{679 \mathrm{~nm}, 1 \mathrm{~cm}}=0.030 \pm 0.005$ after correction by subtraction of the FTO glass absorbance. The absorbance of the photoactive biolayer was estimated as originating from $\sim 5$ monolayers of densely packed PSI complexes [16].

\subsection{Photo-electrochemical measurements}

Photocurrent measurements were performed in a custommade three-electrode photo-electrochemical cell (PEC), as described previously [16]. Briefly, the working electrode (WE) was PSI-FTO glass, the counter electrode (CE) was a platinum wire, and the reference electrode (RE) was $\mathrm{Ag}$ / $\mathrm{AgCl}$ filled with $3 \mathrm{M} \mathrm{KCl}(220 \mathrm{mV}$ vs SHE), connected to the Autolab PGSTAT204 potentiostat. The electrolyte was $30 \mathrm{mM}$ Bis-Tris buffer (pH 7.0) supplemented with $10 \mathrm{mM}$ sodium ascorbate and $200 \mu \mathrm{M}$ dichlorophenolindophenol (DCPIP) as the sacrificial electron donor and mediator, respectively. The illumination source was a 685-nm LED characterized by a $\sim 24-n m$ wide spectral band (FWHM) and light power density of $5.8 \mathrm{~mW} / \mathrm{cm}^{2}$. Open circuit potential (OCP) was measured with a potentiostat in the darkness (typically about $100 \mathrm{mV}$ vs SHE), and then this potential was applied to the WE in all photocurrent experiments to maintain a zero value for the dark current between the WE and CE. The values of IQE were estimated, as described previously in [16].

\subsection{Time-resolved absorption measurements}

The kinetics of photo-oxidized $\mathrm{P}^{+}$decay in PSI complex suspensions was measured at probe wavelength $700 \mathrm{~nm}$ using a transient absorption measuring system, as described in [44]. Briefly, the samples were excited by a $Q$-switched Nd:YAG laser (532 nm; 8 ns FWHM) with different combinations of pulse energy values $(1 \mathrm{~mJ}, 5 \mathrm{~mJ})$ and repetition rates $(0.5 \mathrm{~Hz}, 0.05 \mathrm{~Hz})$ using a perpendicular configuration relative to the probe beam. The probing light source was a continuous $150-\mathrm{W}$ xenon arc lamp equipped with a 700-nm interference filter (10 nm FWHM, FB700-10 Thorlabs). To minimize the actinic effect of the probe light, a shutter was used to enable illumination of the sample by the probe beam only for a short time slightly exceeding the time window of the recorded data. In most experiments, the time window was $~ 54-\mathrm{ms}$ or $\sim 130-\mathrm{ms}$ broad, and to check the presence of faster kinetic components, a $\sim 0.5-\mathrm{ms}$ time window was used. The diameters of the pump and probe beams were $\sim 6 \mathrm{~mm}$ and $\sim 1.5 \mathrm{~mm}$, respectively. The probe light was dispersed using a monochromator which was equipped with an R928 Hamamatsu photomultiplier connected to a digital oscilloscope. Each analyzed kinetic trace was an average of 50 individual traces.

For the time-resolved absorption experiments, all samples were diluted to an optical density $A_{680 \mathrm{~nm}, 1 \mathrm{~cm}} \approx 1$, and measured in the $1 \times 1 \mathrm{~cm}$ quartz cuvette. The PSI particles from Synechocystis and C. reinhardtii were re-suspended in buffer A containing $20 \mathrm{mM}$ Bis-Tris (pH 7.0), $5 \mathrm{mM} \mathrm{MgCl}{ }_{2}$, $5 \mathrm{mM} \mathrm{CaCl}_{2}, 10 \mathrm{mM} \mathrm{NaCl}$ and $0.03 \% \beta$-DM $(v / v)$, whereas the PSI from C. merolae - in buffer B comprising $20 \mathrm{mM}$ HEPES buffer (pH 8.0), $3 \mathrm{mM} \mathrm{CaCl}_{2}$ and $0.03 \% \beta$-DM $(v / v)$. For each sample, measurements were performed firstly without exogenous redox mediators, then with 4-10 mM sodium ascorbate followed by the addition of 4-40 $\mu \mathrm{M}$ DCPIP. The obtained kinetic traces were fitted with the sum of two exponentials function and an offset (const) using the OriginPro program and equation Eq. 1:

$\Delta A(t)=\sum_{i=1}^{n} A_{i} e^{-\frac{t}{\tau_{i}}}+$ const,

where $\tau_{i}$ is the lifetime of $\mathrm{i}$-th component and $A_{i}$ is the amplitude associated with this lifetime.

\subsection{Removing oxygen from the sample}

For oxygen removal, the sample was maintained in a closed cuvette and the space over the solution surface was filled with a flowing stream of argon.

\section{Results and discussion}

\subsection{Photochronoamperometric measurements}

Figure 3A shows representative data of photochronoamperometric curves obtained for the PECs with the biophotoelectrodes functionalized with the PSI complexes isolated from the three different species used in this study. Since the open circuit potential was applied between a $\mathrm{WE}$ and RE, the dark current equaled zero, whereas the illumination of the samples resulted in the generation of negative currents in a $300-700 \mathrm{nA} / \mathrm{cm}^{2}$ range, which disappeared after switching off the light and were similar to 

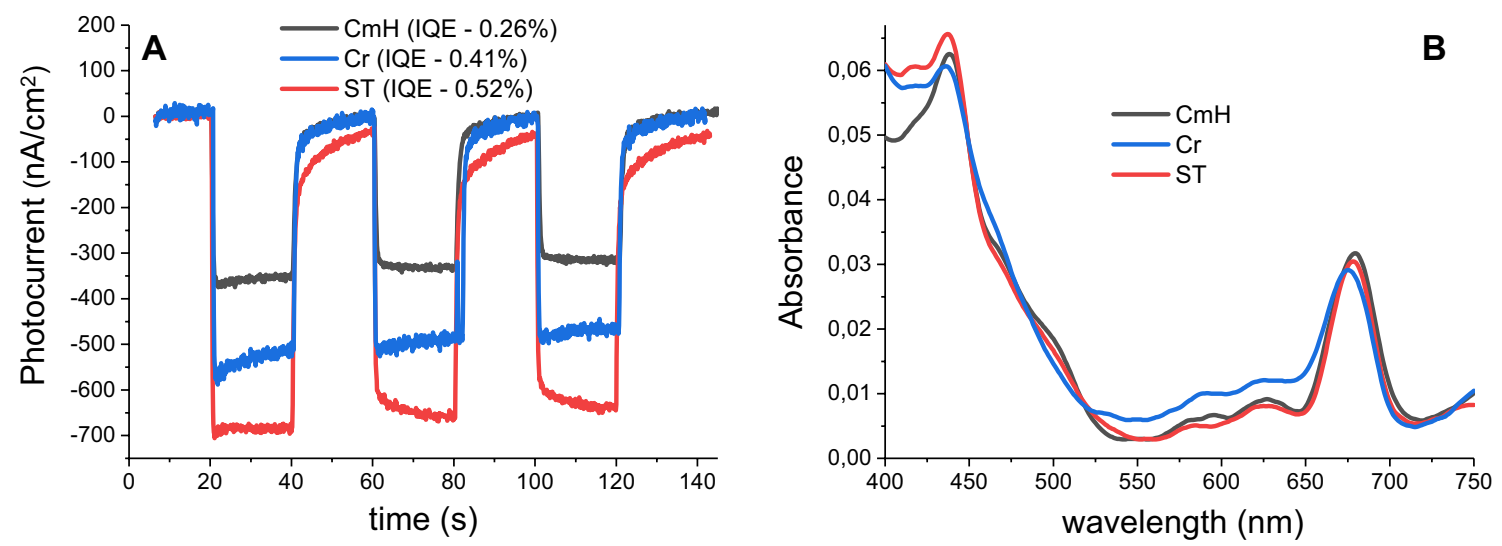

Fig. 3 Photochronoamperometric data (A) and steady-state absorbance spectra (B) of PSI complexes isolated from three species and immobilized on FTO. IQE internal quantum efficiency. (A) Saturating amounts of ascorbate and DCPIP were present

the photocurrent values obtained previously for the cyanobacterial PSI-FTO samples [16]. The negative photocurrent means that the electrons were transferred from the WE (firstly FTO then PSI) through the electrolyte, to the CE. All three biophotoelectrodes contained similar amounts of PSI, as confirmed by similar amplitudes of the steady-state absorption spectra of the PSI-based WE (Fig. 3B). The estimated IQE values ranged from $0.26 \%(\mathrm{Cr})$ to $0.52 \%$ (ST) (Fig. 3A). Such low efficiencies may be due to several reasons, as outlined in the Introduction. One of them could be incomplete charge separation inside the respective RCs followed by an intra-protein charge recombination. To test whether the full charge separation was achieved in PSI particles, we compared charge recombination kinetics in all five PSI samples in aqueous solutions. Our aim was to provide a rational explanation of the limited IQE and photocurrent density values obtained for the photoelectrodes functionalized with the respective PSI bio-photocatalysts.

\subsection{Time-resolved absorption measurements}

To determine the efficiency of charge separation and dynamics of signal decay from the photo-oxidized primary donor in PSI, $P^{+}$, we measured absorption changes at $700 \mathrm{~nm}$, i.e., the wavelength of maximal absorption of the neutral primary donor, $P$, in its ground-state. Photo-oxidation of $P$ causes almost immediate appearance of the negative photobleaching signal followed by its decay caused by reduction of $P^{+}$back to the ground state $(P)$. In principle, reduction of $P^{+}$may occur either due to back ET from one of the intrinsic ET acceptors in the RC or due to ET from an external electron donor. However, the experimental conditions (micro- or milli-second time window) were selected in such a way that in practice, the decay of the $P^{+}$signal was caused mainly by internal charge recombination in the $\mathrm{RC}$, while relatively slow ET from exogenous donor could occur mostly between consecutive excitation laser pulses.

\subsubsection{Results for PSI suspensions without mediators and with saturating concentrations of mediators}

Figure 4 shows the representative $P^{+}$photobleaching decay kinetics in five different PSI aqueous suspensions. For each sample, the measurements were performed in a pure buffer and in the buffer supplemented with external mediators (sodium ascorbate and DCPIP). Ascorbate is an electron donor of rather complex redox chemistry [45], while DCPIP is an ET mediator [46] fully reduced by the excess of ascorbate. The standard redox midpoint potentials of these compounds, $E_{0}$, of $60 \mathrm{mV}$ [45] and $217 \mathrm{mV}$ [47, 48], respectively, confirm that these compounds are suitable for reduction of $P^{+}$primary donor $\left(E_{0}\right.$ of $P^{+} / P$ is $450 \mathrm{mV} \mathrm{[30,}$ 49]), and are therefore commonly used as external electron donors to oxidized $P[36,50,51]$. Previous studies in solution showed that $10 \mathrm{mM}$ ascorbate alone reduces $P^{+}$within tens of seconds [52], whereas the addition of 1-5 $\mu \mathrm{M}$ DCPIP mediator accelerates the $P^{+}$reduction to single seconds [1, 36], while extra $110 \mu \mathrm{M}$ DCPIP further accelerates this process to below $1 \mathrm{~s}$ [52]. Exceptionally fast ET from $2 \mathrm{mM}$ ascorbate to $P^{+}$, of $\sim 200 \mathrm{~ms}$, was reported in ref. [53]. In the mixture of millimolar ascorbate and micromolar DCPIP, reduced species of both compounds prevail. This is because ascorbate is added in its reduced form and DCPIP is practically fully reduced by ascorbate. As discussed in [16], it is not quite clear what species accepts the electrons from PSI-oxidized ascorbate or residual oxidized DCPIP.

3.2.1.1 Initial amplitudes A general observation is that the initial amplitudes of the $\Delta A$ kinetics in all the samples with- 

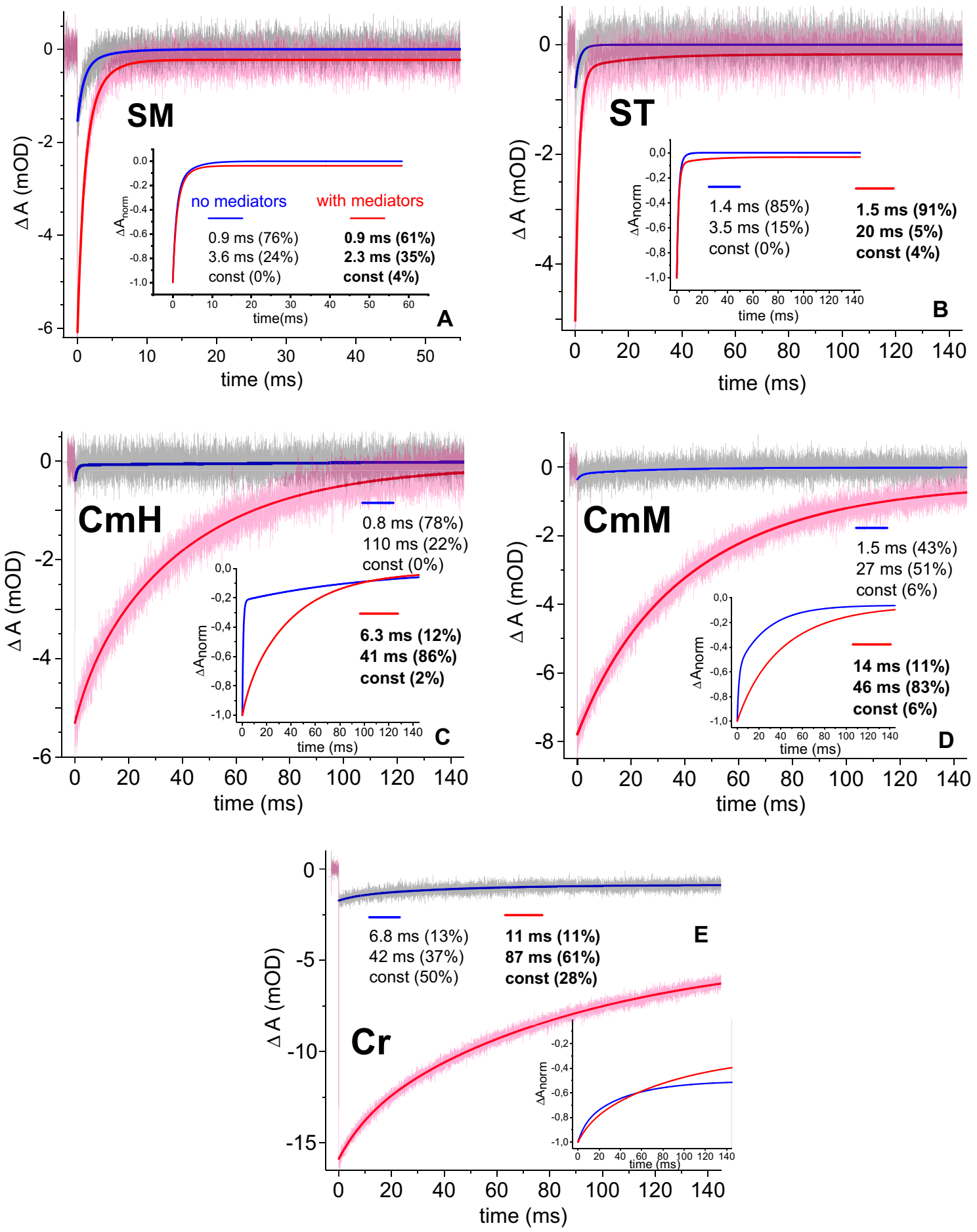

Fig. 4 Representative time-resolved absorption kinetics of five PSI complexes suspended either in buffer only (blue fit curves) or with an addition of saturating concentrations of ascorbate (4-10 $\mathrm{mM})$ and DCPIP $(4-40 \mu \mathrm{M})$ (red fit curves). Main panels - original kinetics and their two-exponential fits, whose parameters are shown in legends. Insets - respective fits normalized to the same initial amplitudes. See Table 1 for further details of experimental conditions and results of data analysis 
Table 1 Selected parameters related to the absorbance changes kinetics (at $700 \mathrm{~nm}$ ) of PSI samples shown in Fig. 4

\begin{tabular}{|c|c|c|c|c|c|c|c|c|c|c|c|c|}
\hline & \multirow[t]{2}{*}{$\begin{array}{l}\Delta \mathrm{A}_{0} \\
(\mathrm{mOD})\end{array}$} & \multirow[t]{2}{*}{$\Delta \mathrm{A}_{0 \text { rel }}$} & \multicolumn{4}{|c|}{$\begin{array}{l}\tau_{\mathrm{i}}(\mathrm{ms}) \\
\mathrm{A}_{\mathrm{i}} \\
\end{array}$} & \multirow[t]{2}{*}{$\begin{array}{l}\text { const } \\
(\%)\end{array}$} & \multirow[t]{2}{*}{$\begin{array}{l}\tau_{\mathrm{av}} \\
(\mathrm{ms})\end{array}$} & \multirow[t]{2}{*}{$\begin{array}{l}\tau_{\text {out }} \\
(\mathrm{ms})\end{array}$} & \multirow[t]{2}{*}{$\mathrm{E}_{\mathrm{exc}}$} & \multirow[t]{2}{*}{$\begin{array}{l}\mathrm{f} \\
(\mathrm{Hz})\end{array}$} & \multirow[t]{2}{*}{$\begin{array}{l}\text { [asc] } \\
{[\mathrm{DCPIP}]}\end{array}$} \\
\hline & & & $\mathrm{P}^{+} \mathrm{F}_{\mathrm{X}}$ & $\rightarrow \mathrm{PF}_{3}$ & $\mathrm{P}^{+} \mathrm{F}_{\mathrm{A}}$ & $/ \mathrm{B}^{-} \rightarrow \mathrm{PF}_{\mathrm{A} / \mathrm{B}}$ & & & & & & \\
\hline \multicolumn{13}{|l|}{$S M$} \\
\hline Buffer only & 1.5 & $25 \%$ & $\begin{array}{l}0.9 \\
76 \%\end{array}$ & $\begin{array}{l}3.6 \\
24 \%\end{array}$ & - & - & - & 1.5 & - & $1 \mathrm{~mJ}$ & 0.5 & - \\
\hline+ mediators & 6.0 & $100 \%$ & $\begin{array}{l}0.9 \\
61 \%\end{array}$ & $\begin{array}{l}2.3 \\
35 \%\end{array}$ & - & - & - & 1.4 & 34 & $1 \mathrm{~mJ}$ & 0.5 & $\begin{array}{l}10 \mathrm{mM} \\
4 \mu \mathrm{M}\end{array}$ \\
\hline \multicolumn{13}{|l|}{$S T$} \\
\hline Buffer only & 0.8 & $16 \%$ & $\begin{array}{l}1.4 \\
85 \%\end{array}$ & $\begin{array}{l}3.5 \\
15 \%\end{array}$ & - & - & $-\overline{0}$ & 1.7 & - & $1 \mathrm{~mJ}$ & 0.5 & - \\
\hline+ mediators & 5.0 & $100 \%$ & - & $\begin{array}{l}1.5 \\
91 \%\end{array}$ & $\begin{array}{l}\mathbf{2 0} \\
\mathbf{5} \%\end{array}$ & - & - & 2.4 & 58 & $1 \mathrm{~mJ}$ & 0.5 & $\begin{array}{l}10 \mathrm{mM} \\
40 \mu \mathrm{M}\end{array}$ \\
\hline \multicolumn{13}{|l|}{$\mathrm{CmH}$} \\
\hline Buffer only & 0.4 & $8 \%$ & - & $\begin{array}{l}0.8 \\
78 \%\end{array}$ & $\begin{array}{l}110 \\
22 \%\end{array}$ & - & $-\overline{0}$ & 25 & - & $1 \mathrm{~mJ}$ & 0.5 & - \\
\hline+ mediators & 5.3 & $100 \%$ & - & & $\begin{array}{l}6.3 \\
12 \%\end{array}$ & $\begin{array}{l}41 \\
86 \%\end{array}$ & $\overline{2} \%$ & 37 & 1900 & $1 \mathrm{~mJ}$ & 0.5 & $\begin{array}{l}10 \mathrm{mM} \\
40 \mu \mathrm{M}\end{array}$ \\
\hline \multicolumn{13}{|l|}{ I } \\
\hline Buffer only & 0.4 & $5 \%$ & - & $\begin{array}{l}1.5 \\
43 \%\end{array}$ & $\begin{array}{l}27 \\
51 \%\end{array}$ & - & $\begin{array}{l}- \\
6 \%\end{array}$ & 16 & - & $5 \mathrm{~mJ}$ & 0.5 & - \\
\hline+ mediators & 7.8 & $100 \%$ & - & - & $\begin{array}{l}14 \\
11 \%\end{array}$ & $\begin{array}{l}46 \\
83 \%\end{array}$ & - & 42 & 660 & $1 \mathrm{~mJ}$ & 0.5 & $\begin{array}{l}4 \mathrm{mM} \\
40 \mu \mathrm{M}\end{array}$ \\
\hline \multicolumn{13}{|l|}{$\mathrm{Cr}$} \\
\hline Buffer only & 1.7 & $11 \%$ & - & & $\begin{array}{l}6.8 \\
13 \%\end{array}$ & $\begin{array}{l}42 \\
37 \%\end{array}$ & $\begin{array}{l}- \\
50 \%\end{array}$ & 33 & - & $1 \mathrm{~mJ}$ & 0.05 & - \\
\hline+ mediators & 16 & $100 \%$ & - & - & $\begin{array}{l}11 \\
11 \%\end{array}$ & $\begin{array}{l}87 \\
61 \%\end{array}$ & - & 75 & 190 & $1 \mathrm{~mJ}$ & 0.05 & $\begin{array}{l}4 \mathrm{mM} \\
40 \mu \mathrm{M}\end{array}$ \\
\hline
\end{tabular}

$\Delta A_{0}$ - initial $\Delta A$ amplitude just after excitation; $\Delta A_{\text {0rel }}=\frac{\Delta A_{0}}{\Delta A_{0} \text { (withmediators) }} 100 \%$ - initial $\Delta A$ amplitude just after excitation, relative to the initial $\Delta A$ amplitude just after excitation in the respective experiment with mediators; $\tau_{i}, A_{i}$ const - bi-exponential fit parameters; $\tau_{\text {av }}-$ an average charge recombination time constant estimated from Eq. 3; $\tau_{\text {out }}$ - time constant of ET outside PSI estimated from Eq. 2; $E_{\text {exc }}$ - excitation energy; $f$-excitation frequency; [asc], [DCPIP] - concentrations of ascorbate and DCPIP, respectively out external electron donors (ascorbate and DCPIP) in solution are much smaller than those of the respective samples with the addition of these donors (see Fig. 4 and Table 1 for exact values). This difference is caused by the quasi-permanent oxidation of $P$ to $P^{+}$, in the absence of mediators, in the majority of PSI RCs, due to the repetitive excitation and (to a lesser extent) probe flashes upon accumulation of tens of kinetic traces to be averaged for an improved signal-to-noise ratio. Such a situation is possible only if charge separation in at least a fraction of PSI particles is followed by ET from the intrinsic terminal PSI electron acceptor to an external electron acceptor present in the solution. Even if the probability of electron escape from PSI after a single excitation flash is low, after many laser pulses (at 0.5 or $0.05 \mathrm{~Hz}$ frequency), a constant fraction of "optically inactive" PSI complexes (containing $\mathrm{P}^{+}$and no electron on the terminal acceptor) is formed. Such a situation is presented schematically in Fig. 5A and $\mathrm{C}$ with optically active and inactive PSI fractions shown. In the solutions without mediators, the optically active RCs are unable to transfer electrons outside PSI (or do this too slowly) and the ground state of the ETC is recovered by a charge recombination before each subsequent laser flash. This inability to transfer electrons outside PSI is reasonable since in the absence of external mediators such ET seems impossible. However, the situation is inverse for most complexes which become optically inactive (Fig. 5A and C_- "inactive fractions"). We propose that they become optically inactive due to electron "leakage" (transfer) outside PSI, leaving the protein with a long-lived (compared to intervals between the flashes), and thus, optically invisible $P^{+}$state (states $P^{+} F_{X}$ in Fig. $5 \mathrm{~A}$ and $P^{+}\left(\mathrm{F}_{\mathrm{A}} / \mathrm{F}_{\mathrm{B}}\right)$ in Fig. 5C). The existence of ET outside PSI is surprising since there were no external mediators added to the solution. We propose that even without the intentional addition of an external electron acceptor, some compounds present in the solution may play such a role. One such compound could be oxygen dissolved in the solution. In a control experiment, oxygen was removed from the solution. However, even 
No mediators

Active fraction, $\sim 20 \%$

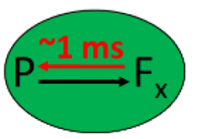

Inactive fraction, $80 \%$

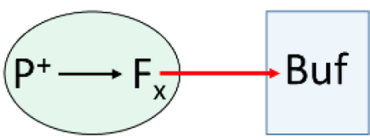

\section{With mediators}

\section{Synechocystis}

B

Active fraction, $100 \%$

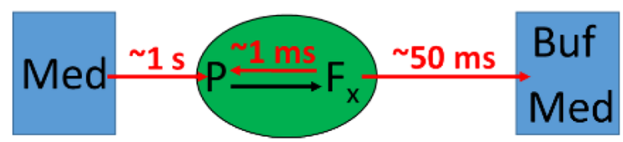

\section{C. merolae, C. reinhardtii}

C

Active fraction, $\sim 10 \%$

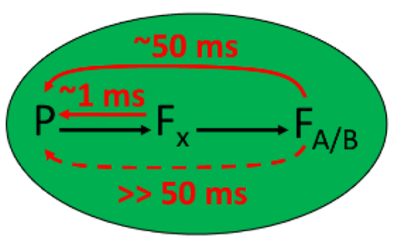

Inactive fraction, $\sim 90 \%$

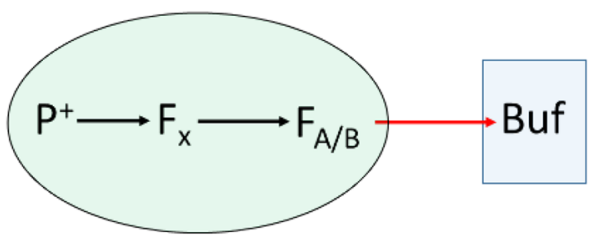

D

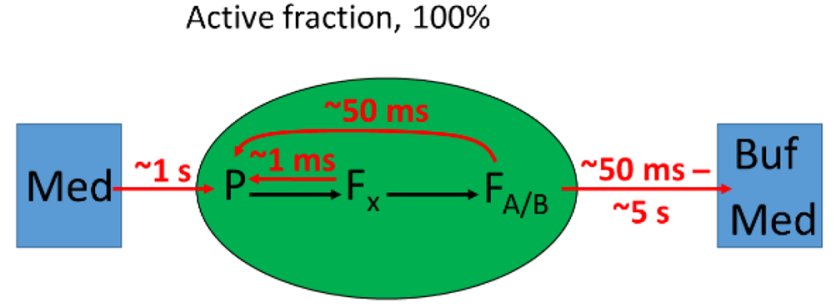

Fig. 5 Scheme of major electron transfer reactions inside and outside PSI complexes from Synechocystis $(\mathbf{A}, \mathbf{B})$ and C. merolae/C. reinhardtii $(\mathbf{C}, \mathbf{D})$. Panels $\mathbf{A}$ and $\mathbf{C}$ - no ET mediators were present in the buffer solution; panels $\mathbf{B}$ and $\mathbf{D}$-saturating amount of ET mediators were present in the buffer solution. Black arrows depict fast forward ET steps inside PSI, not resolved in our experiments. Red arrows depict ET steps responsible for the initial amplitudes and decay rates of the $P^{+}$kinetic traces (the $\sim 1-\mathrm{ms}$ back ET from $\mathrm{F}_{\mathrm{x}}{ }^{-}$to $\mathrm{P}^{+}$in panel D

then, the majority of PSI complexes remained optically inactive when no mediators were added (data not shown). This control experiment suggested that oxygen dissolved in the solution was not the species responsible for electron uptake from PSI. We hypothesize that electrons from PSI can be transferred to the molecules of the buffer. This seems justified since the redox midpoint potential of all PSI electron acceptors is very negative (see Fig. 2). In principle, the postulated presence of the redox molecules in the solution without ascorbate and DCPIP should be detectable by was not directly observed for $\mathrm{Cm}$ and $\mathrm{Cr}$ but was taken from the literature $[36,50]$ and is supposed to affect the 6-14 ms recombination components shown in Table 1). Dark green ovals - PSI complexes contributing to the $P^{+} \Delta A$ signal. Light green ovals - PSI complexes not contributing to the $P^{+} \Delta A$ signal, due to quasi-permanent oxidation of $P$. Blue rectangles - solution of buffer (Buf) with or without ET mediators (Med)

performing cyclic voltammetry with the electrolyte alone. Alternatively, our procedure of replacement of oxygen with argon was not efficient enough, and residual oxygen species could still accept photo-generated electrons from a sub-pool of PSI particles.

After the addition of ascorbate and DCPIP, the initial amplitude of $\Delta A$ kinetics increased 4-20-fold, depending on the sample (Fig. 4 and Table 1). This effect reveals that between excitation flashes, DCPIP reduces $P^{+}$to $P$ and recovers the full capacity of the system to undergo 
photooxidation of $P$. By adjusting the concentration of mediators and excitation frequency we confirmed that the initial amplitudes for the samples containing mediators (shown in Fig. 4) were maximal (see below), indicating that all the analyzed PSI complexes were active (see Fig. 5B and D) and that the applied concentrations of mediators were saturating for a full PSI RC turnover.

\subsubsection{Kinetics of $P^{+}$decay in PSI from Synechocystis sp.} PCC 6803 monomers and trimers The kinetics of $P^{+}$decay for all the samples is different when measured with and without redox mediators (Fig. 4). Since the initial $\Delta A$ amplitudes are much bigger in the first case, the decay kinetics recorded under these conditions (with external mediators) are more representative for the whole populations of PSI particles under study and therefore, for each of the samples, we analyzed them firstly. Subsequently, we analyzed the kinetics originating from optically active PSI sub-pools in the absence of external mediators.

The $P^{+}$decay kinetics for all the samples (see Fig. 4) were fitted with a bi-exponential decay function plus an offset (const; Eq. 1). The fit parameters are shown both in Fig. 4 and in Table 1. It is clear from these data that the samples under study are characterized by very different $P^{+}$decay kinetics.

For the Synechocystis SM and ST PSI samples, the decay kinetics occurs mostly within $\sim 1-2 \mathrm{~ms}$ (Fig. 4A and B), which is a lifetime value characteristic for $P^{+} F_{X}{ }^{-} \rightarrow P F_{X}$ charge recombination $[36,50]$. For the SM sample, no slower phase was observed while for the ST suspension, 5\% of the initial signal decays with a 20-ms time constant, which is assigned to $P^{+}\left(F_{A} / F_{B}\right)^{-} \rightarrow P\left(F_{A} / F_{B}\right)$ charge recombination $[36,50]$. We conclude that for almost entire population of Synechocystis PSI particles in solution, the photo-excited electron does not reach the terminal electron acceptor $\left(F_{A} / F_{B}\right)$. This observation indicates that the cyanobacterial PSI particles, which were stored at $-20^{\circ} \mathrm{C}$ in a buffer devoid of glycerol, were most likely structurally perturbed, possibly due to detachment of the peripheral PsaC subunit coordinating $\left(F_{A} / F_{B}\right)$ clusters. Under such proposed structural perturbation of cyanobacterial PSI, the $F_{X}$ cluster would become the final acceptor. In the context of the photovoltaic application of such perturbed PSI preparations, it is important to emphasize that $F_{X}{ }^{-}$may transfer electrons outside the protein. This is evidenced by a significant decrease in the initial $\Delta A$ amplitude in the absence of external mediators, as discussed above (Fig. 4). Consequently, a non-decaying kinetic component, const, in Eq. 1 is interpreted as a result of electron escape from $F_{X}{ }^{-}$to the electrolyte in a process that competes with internal $P^{+} F_{X}{ }^{-} \rightarrow P F_{X}$ charge recombination, and which promotes a long-lived (practically non-decaying) state, $P^{+} F_{X}$. Under such assumption, it is possible to estimate the time constant of ET outside PSI, $\tau_{\text {out }}$, from the fit parameters according to Eq. 2: $\tau_{\mathrm{out}}=\tau_{\mathrm{av}} \frac{A_{1}+A_{2}}{\text { const }}$

where amplitudes $A_{1}, A_{2}$, and const are defined in Eq. 1, and $\tau_{\mathrm{av}}$ is an average charge recombination time constant given by the formula in Eq. 3:

$\tau_{\mathrm{av}}=\frac{\tau_{1} A_{1}+\tau_{2} A_{2}}{A_{1}+A_{2}}$.

From Eq. 2, ET outside PSI is estimated at $\sim 50 \mathrm{~ms}$ for both SM (34 ms) and ST (58 ms) (see Tab. 1), which is an order of magnitude faster than the kinetics of ET outside of PSI (ET "leakage") measured for the remaining PSI particles in solution (see below).

At this point, one should note that strictly speaking the offset is only an approximate measure of the efficiency of electron escape from PSI to the electrolyte (in all the samples under study) and may underestimate this efficiency in all the samples under study. This statement may be justified in the following way. Equation 2 may be rewritten in the form:

$\frac{k_{\text {out }}}{k_{\text {av }}}=\frac{\text { const }}{A_{1}+A_{2}}$,

where $k_{\text {out }}=1 / \tau_{\text {out }}$ and $k_{\mathrm{av}}=1 / \tau_{\mathrm{av}}$ is the average rate of charge recombination. However, if one takes into account a possibility that the decay kinetics may be, to some extent, affected by ET from the exogenous donor to $P^{+}$, both const and the sum $A_{1}+A_{2}$ may contain contributions from this process. On the other hand, such ET leaves PSI in the state $P\left(F_{x} / F_{A / B}\right)^{-}$, subsequently oxidized by the mediators to the state $P\left(F_{x} / F_{A / B}\right)$. Thus, the ET from the exogenous donor to $P^{+}$leads to ET from PSI to the electrolyte. This way, not only the const parameter but also a part of the sum $A_{1}+A_{2}$ may contribute to the escape of electrons from PSI to the electrolyte, and the sole const parameter may underestimate this efficiency. Moreover, if one takes into account the reduction of $P^{+}$by the exogenous mediator, the following differential equation properly describes the temporal evolution of concentration $\left[P^{+}\right]$of the RCs with $P^{+}$, and thus the measured kinetics:

$\frac{d\left[P^{+}\right]}{d t}=-\left(k_{\mathrm{out}}^{\prime}+k_{\mathrm{av}}^{\prime}+k_{P}\right)\left[P^{+}\right]$,

where $k_{P}$ is the rate of ET from the exogenous donor to $P^{+}$, whereas $k_{\text {out }}^{\prime}$ and $k_{\text {av }}^{\prime}$ may be different from their counterparts in Eq. 4 and satisfy the equation:

$\frac{k_{\mathrm{out}}^{\prime}}{k_{\mathrm{av}}^{\prime}+k_{P}}=\frac{C_{\mathrm{out}}}{A_{1}+A_{2}+C_{P}}$,

where $C_{\text {out }}$ and $C_{P}$ are the contributions to the const parameter, const $=C_{\text {out }}+C_{P}$, originating from the RCs in which 
ET from the electrolyte to $P^{+}$follows $\left(C_{\text {out }}\right)$ or precedes $\left(C_{P}\right)$ electron escape from PSI to the electrolyte. By comparing Eqs. 4 and 6, one can see that $k_{\text {out }} \leq k_{\text {out }}$ and thus, the neglect of ET from the electrolyte to $P^{+}$may overestimate the rate $k_{\text {out }}$. Consequently, $\tau_{\text {out }}$ should be treated as an approximate time of electron escape from PSI. Conversly, when $k_{\text {av }}^{\prime}>>k_{P}$, Eq. 6 reduces to Eq. 4 .

The SM and ST PSI complexes re-suspended in a buffer without exogenous mediators show generally similar $P^{+}$ decay kinetics to the respective decay characteristics for the complexes in a buffer with mediators (Fig. 4A, B and Table 1). The most significant difference is the lack of offset in the former case. This indicates that in the fractions of complexes exhibiting the $\Delta \mathrm{A}$ signals in solution without mediators, ET outside PSI does not compete with the charge recombination. These fractions amount to $25 \%$ and $16 \%$ for SM and ST samples, respectively, as estimated from the initial $\Delta \mathrm{A}$ signals. We conclude that in these fractions, ET outside PSI does not occur for some, likely structural, reasons. In the remaining fractions (of $75 \%$ and $84 \%$ for SM and ST, respectively), ET outside PSI does occur and the resulting long-lived state $P^{+} F_{X}$ makes them undetectable in transient absorption experiments at $700 \mathrm{~nm}$. Thus, the cyanobacterial SM and ST PSI complexes show a certain degree of heterogeneity regarding the occurrence of ET outside the RC.

\subsubsection{Kinetics of $P^{+}$decay in PSI from C. merolae The} kinetics of the $P^{+}$decay in two preparations of PSI from C. merolae is dominated by a $\sim 45-\mathrm{ms}$ phase $(\sim 85 \%$; Fig. $4 \mathrm{C}, \mathrm{D}$ and Table 1). This phase was assigned to the $P^{+}\left(F_{A} / F_{B}\right)^{-} \rightarrow P\left(F_{A} / F_{B}\right)$ charge recombination [36, 50]. A smaller fraction of $11-12 \%$ decays within $\sim 6-14 \mathrm{~ms}$ and was also assigned to $P^{+}\left(F_{A} / F_{B}\right)^{-} \rightarrow P\left(F_{A} / F_{B}\right)$ charge recombination with a possible admixture of $P^{+} F_{x}{ }^{-} \rightarrow P F_{x}$ charge recombination [50]. In $\mathrm{CmH}$, the offset amounts to $2 \%$, whereas in $\mathrm{CmM}-6 \%$. The interpretation of this component is the same as in the case of cyanobacterial SM and ST PSI samples: it is mainly caused by ET outside PSI which competes with charge recombination inside the RC. However, in contrast to cyanobacterial PSI, ET outside $C$. merolae PSI particles occurs from $\left(F_{A} / F_{B}\right)^{-}$rather than from $F_{X}{ }^{-}$. We conclude that in both $\mathrm{CmH}$ and $\mathrm{CmM}$ samples, most of the red algal PSI particles (>90\%) support the full forward ET from the primary electron donor to the terminal electron acceptor, $\left(F_{A} / F_{B}\right)$. At most, only a few percent of red algal PSI complexes (more in $\mathrm{CmH}$ than in $\mathrm{CmM}$ ) show incomplete charge separation leading to the formation of $P^{+} F_{X}{ }^{-}$species. The ET outside PSI is about three times more efficient in the case of $\mathrm{CmM}$ than in $\mathrm{CmH}$ PSI particles. This is reflected by about a threefold smaller value of the time constant $\tau_{\text {out }}$ measured for the CmM sample (660 ms) compared to the $\mathrm{CmH}$ counterpart (1900 ms). Interestingly, both time constants are at least an order of magnitude larger than the corresponding values obtained for the cyanobacterial PSI samples. This explains why, despite much slower average charge recombination in $C$. merolae samples, the efficiency of ET outside PSI was not very much different in both species (4\% in cyanobacteria and $2-6 \%$ in C. merolae). In the context of photovoltaic applications, the faster ET outside PSI observed for the putatively truncated PSI from Synechocystis together with its preserved activity both in the solution and upon immobilization on the electrode surface, indicates potential advantages of using such disturbed photoactive structures. First, the $F_{X}{ }^{-}$cofactor is more easily accessible to the external electron acceptor in the PSI protein devoid of PsaC compared to $\left(F_{A} / F_{B}\right)^{-}$terminal acceptor (as indicated by smaller values of $\tau_{\text {out }}$ ). Second, $F_{X}$ is characterized by a more negative redox potential than $\left(F_{A} / F_{B}\right)^{-}$(see Fig. 1), which further facilitates the reduction of the external redox carriers.

The initial amplitudes of the $P^{+}$decay kinetics in $C$. merolae PSI particles decrease in the solution without mediators to only $5-8 \%$ (Table 1). This is less than in the case of cyanobacterial samples (16-25\%). Observation of the $\Delta A$ signals under these conditions again indicates that the small fraction of red algal PSI complexes are unable to transfer electrons outside PSI. The average decay lifetime, $\tau_{\mathrm{av}}$, without mediators is accelerated (compared to the samples with mediators). This is because, in both samples measured without mediators, a large fraction of the signal decays within $\sim 1 \mathrm{~ms}$ (78\% in $\mathrm{CmH}$ and $43 \%$ in $\mathrm{CmM}$ ), indicating a large contribution from $P^{+} F_{X}{ }^{-} \rightarrow P F_{X}$ recombination and disturbance in complete charge separation. Another large fraction decays within $~ 30-110 \mathrm{~ms}$ assigned to $P^{+}\left(F_{A} / F_{B}\right)^{-} \rightarrow P\left(F_{A} / F_{B}\right)$ charge recombination. In the case of $\mathrm{CmM}$, the minor fraction of the signal $(6 \%)$ does not decay in the experimental time window and is assigned to particularly slow charge recombination in disturbed proteins unable to transfer electrons outside PSI. Resolution of as many as three phases in the minor fraction of PSI complexes giving the signals even in the absence of mediators reveals heterogeneity of this fraction. In the case of $\mathrm{CmH}$, const $=0$, similarly as in the respective measurements of the cyanobacterial PSI samples.

3.2.1.4 Kinetics of $P^{+}$decay in PSI from C. reinhardtii We did not observe any 1-2 ms component in $P^{+}$decay in PSI from $C$. reinhardtii (Table 1). Still, its kinetics is biexponential with a major phase (61\%) decaying within $87 \mathrm{~ms}$ and a minor one $(11 \%)$ decaying within $11 \mathrm{~ms}$. The latter phase may contain a contribution from the particularly slow $P^{+} F_{X}{ }^{-} \rightarrow P F_{X}$ recombination process. However, we conclude that the vast majority of PSI particles support the full ET from the primary donor $P$ to the terminal acceptor $F_{A} / F_{B}$. The observed millisecond kinetics are essentially due to $P^{+}\left(F_{A} / F_{B}\right)^{-} \rightarrow P\left(F_{A} / F_{B}\right)$ charge recombination. Impor- 
tantly, the non-decaying phase in this sample was particularly large (28\%), much larger than for the other analyzed samples (2-6\%). This difference is both due to a relatively fast ET outside PSI ( $\tau_{\text {out }}=190 \mathrm{~ms}$, Table 1 ; faster than for C. merolae $)$ and particularly slow $P^{+}\left(F_{A} / F_{B}\right)^{-} \rightarrow P\left(F_{A} / F_{B}\right)$ charge recombination $\left(\tau_{\mathrm{av}}=75 \mathrm{~ms}\right)$, roughly twice slower than in $C$. merolae $\left(\tau_{\mathrm{av}}=\sim 40 \mathrm{~ms}\right)$. The fast ET outside PSI indicates that $\left(F_{A} / F_{B}\right)$ acceptor is more easily accessible in $C$. reinhardtii than in $C$. merolae. This may be related to the lack of LHCI complexes in the investigated strain of $C$. reinhardtii PSI.

Similarly to the other PSI samples under study, in the sample without mediators, the initial amplitude largely decreased compared to the sample with mediators ( tenfold; Table 1), and the $P^{+}$decay kinetic was accelerated (Fig. 4E; the lifetimes $6.8 \mathrm{~ms} \mathrm{(13 \% )} \mathrm{and} 42 \mathrm{~ms} \mathrm{(37 \% )}$ give the average value $\tau_{\mathrm{av}}=33 \mathrm{~ms}$ that is about twice smaller than $75 \mathrm{~ms}$ estimated for the sample with mediators). Both $6.8-\mathrm{ms}$ and $42-\mathrm{ms}$ phases were assigned to $P^{+}\left(F_{A} / F_{B}\right)^{-} \rightarrow P\left(F_{A} / F_{B}\right)$ recombination with a possible contribution from $P^{+} F_{X}{ }^{-} \rightarrow P F_{X}$ recombination in the former phase. A large offset (50\%) was assigned to a small fraction (relatively to the total population: $50 \%$ out of $11 \%$ give $5.5 \%$ ) of particularly slowly recombining PSI RCs unable to transfer electrons outside the protein.

\subsubsection{Comprehensive survey of the $P^{+}$decay kinetics}

In addition to the measurements of $P^{+}$decay kinetics in PSI solutions without mediators and with saturating concentrations of ascorbate and DCPIP, the samples were also prepared with the ascorbate sacrificial donor only. Moreover, we compared systematically the results obtained for the samples with all the three compositions of mediators and with all five types of PSI complexes at two excitation energies ( 1 and $5 \mathrm{~mJ})$, two excitation frequencies $(0.5$ and $0.05 \mathrm{~Hz})$, and two concentrations of DCPIP ( 4 and $40 \mu \mathrm{M})$. A comprehensive comparison is presented in Fig. 6. The individual experimental conditions are labeled by numbers 1-12 shown on the horizontal axes of all the panels in Fig. 6, and their description is provided in Table 2. Overall, the experimental conditions were ordered in such a way, identical for all the samples, that the higher is the number of the experiment, the more efficient is the chemical reduction of the photo-oxidized primary donor $P^{+}$. The most meaningful are dependencies of initial amplitudes vs. reduction power of experimental conditions, while the dependencies of the remaining quantities are less regular due to a limited signalto-noise ratio of the raw kinetics data.

3.2.2.1 Initial amplitudes of the $P^{+}$decay kinetics Comparison of the initial amplitudes of the $P^{+}$decay kinetics $\left(-\Delta A_{0}\right)$ for all the five PSI preparations shows a general sys- tematic dependence (Fig. 6, the first column of panels: A, E, $\mathrm{I}, \mathrm{M}, \mathrm{Q})$ : the higher is the number indicating the experimental conditions (1-12), the larger the initial amplitude (except for experiments 10-12 for SM-Fig. 6A; see below). However, the dynamics of this dependence are various for different samples.

The first three experimental condition sets (Fig. 6, experiments 1-3; in gray) refer to the samples without mediators. For all the samples, the five-fold increase in the excitation energy, from $1 \mathrm{~mJ}$ (experiment 1, compare Table 2) to $5 \mathrm{~mJ}$ (experiment 2) caused only a slight increase in the signal. This small effect shows that $1 \mathrm{~mJ}$ is a value that almost saturates the signal. On the other hand, for all the samples, decreasing the excitation frequency from $0.5 \mathrm{~Hz}$ (experiment 1 ) to $0.05 \mathrm{~Hz}$ (experiment 3) exerts a stronger effect on the signal than the increased excitation energy (experiment 2). This shows that the signal recorded at $0.5 \mathrm{~Hz}$ is limited by a slow relaxation of the sample occurring on a time scale of seconds. The likely origin of this relaxation is the particularly slow charge recombination in a small fraction of disturbed PSI particles being in the state $P^{+} F_{X}{ }^{-}$(cyanobacteria) and/or $P^{+}\left(F_{A} / F_{B}\right)^{-}$(remaining species), or a slow electron donation from the buffer solution to $P^{+}$in a fraction of PSI being in the state $P^{+} F_{X}$ (cyanobacteria) or $P^{+}\left(F_{A} / F_{B}\right)$ (remaining species). Since there were no mediators in the solution in experiments $1-3$, the latter possibility seems less likely.

The addition of ascorbate alone at low excitation energy $(1 \mathrm{~mJ})$ and high repetition rate $(0.5 \mathrm{~Hz})$ (experiment 4 ; in yellow) increased the signal (compare to experiments 1-3), although not to a maximum level (compare to the following experiments; see below). Apparently, ascorbate reduces $P^{+}$by a direct electron donation to the primary donor [52]. This effect was stronger when $10 \mathrm{mM}$ ascorbate was added (Fig. 6A, E, I) compared to when $4 \mathrm{mM}$ ascorbate was used (Fig. 6M, Q) as expected. An increase in the excitation energy to $5 \mathrm{~mJ}$ (experiment 5), similarly as for the samples without mediators, only slightly $(<20 \%)$ increased the signal. On the other hand, a decrease in the excitation frequency (experiment 6) again caused a strong increase in the $-\Delta A_{0}$ signal, yet not to the highest possible level that was observed in the subsequent experiments (see below). Evidently, the millimolar concentration of ascorbate alone is not enough to recover fully the ground state of the photo-oxidized primary donor, $P$, within $20 \mathrm{~s}$.

Upon addition of $4 \mu \mathrm{M}$ DCPIP to the samples containing ascorbate at $E_{\text {exc }}=1 \mathrm{~mJ}$ and $f=0.5 \mathrm{~Hz}$ (experiment 7; in light blue), an almost saturating effect was observed for the $-\Delta A_{0}$ signals from the cyanobacterial samples (Fig. 6A and E) but not for the remaining species (Fig. 6M and Q). Most likely, the difference was caused by easier access of DCPIP to $P^{+}$in the cyanobacterial PSI. The increase in the excitation energy to $5 \mathrm{~mJ}$ (experiment 8 ) only slightly increased the signal in 

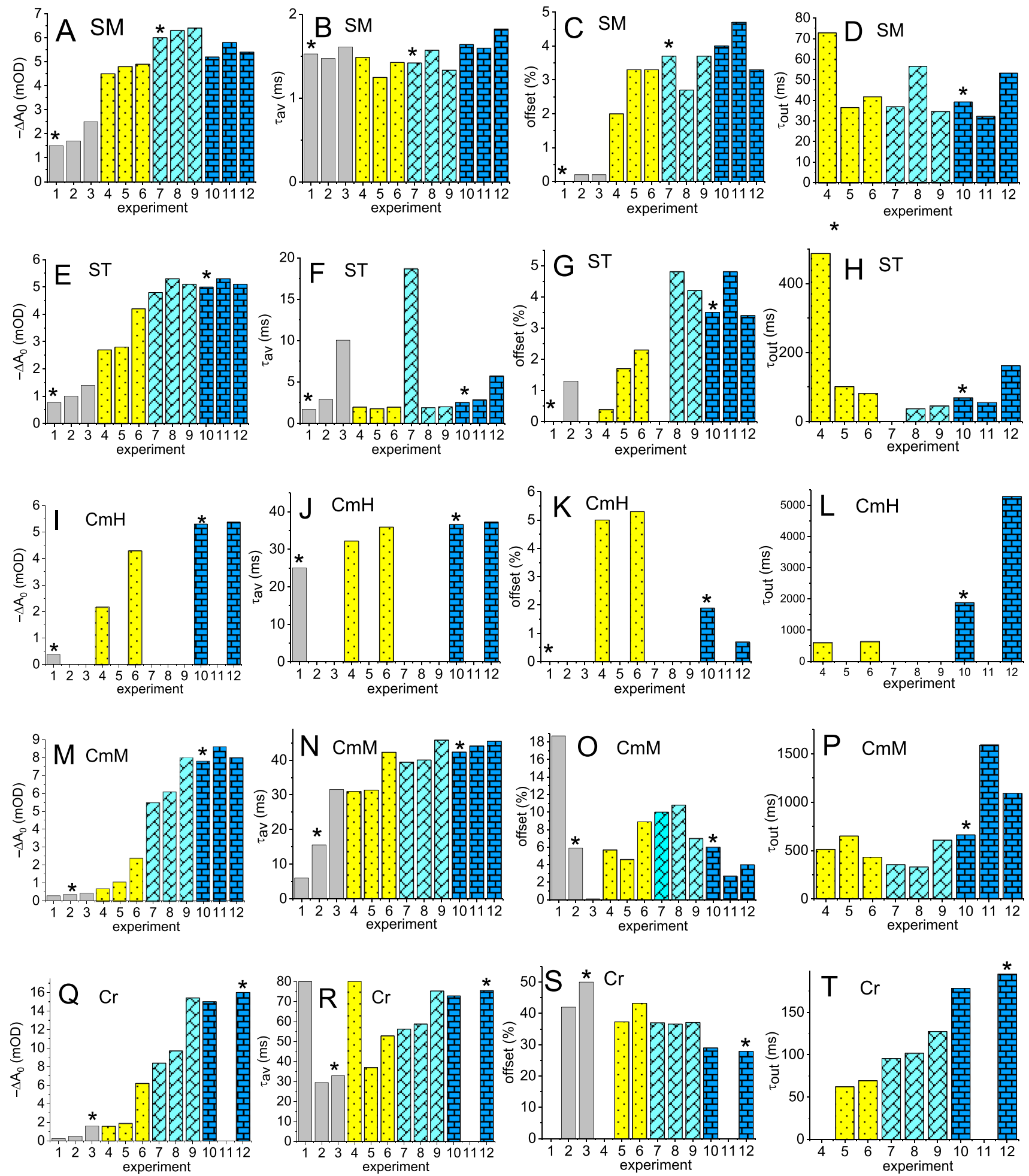

Fig. 6 Comparison of the results of the $P^{+}$decay measurements performed under a range of experimental condition (represented by numbers 1-12 shown under the abscissa). The experimental conditions are explained in Table 2. The five rows of panels correspond to the five types of PSI complexes. The stars indicate the data correspond-

ing to the kinetics presented in Fig. 4. $\Delta A_{0}=\Delta A(t=0)$, where $\Delta A(t)$ and offset (const) are the quantities from Eq. 1. $\tau_{\text {out }}$ and $\tau_{\text {av }}$ are the quantities from Eqs. 2 and 3, respectively. Note that for $\mathrm{CmH}$ and $\mathrm{Cr}$ PSI complexes, some experimental conditions were omitted 
Table 2 Experimental conditions of the $P^{+}$decay measurements

\begin{tabular}{lllll}
\hline $\begin{array}{l}\text { Experiment } \\
\text { number }\end{array}$ & {$[\mathrm{asc}]^{*}(\mathrm{mM})$} & {$[\mathrm{DCPIP}](\mu \mathrm{M})$} & $E_{\mathrm{exc}}(\mathrm{mJ})$ & $f(\mathrm{~Hz})$ \\
\hline 1 & - & - & 1 & 0.5 \\
2 & - & - & 5 & 0.5 \\
3 & - & - & 1 & 0.05 \\
4 & 10 & - & 1 & 0.5 \\
5 & 10 & - & 5 & 0.5 \\
6 & 10 & - & 1 & 0.05 \\
7 & 10 & 4 & 1 & 0.5 \\
8 & 10 & 4 & 5 & 0.5 \\
9 & 10 & 4 & 1 & 0.05 \\
10 & 10 & 40 & 1 & 0.5 \\
11 & 10 & 40 & 5 & 0.5 \\
12 & 10 & 40 & 1 & 0.05 \\
\hline
\end{tabular}

* In the case of $\mathrm{CmM}$ and $\mathrm{Cr}$ samples, $4 \mathrm{mM}$ ascorbate was used instead of $10 \mathrm{mM}$ after confirming that the former ascorbate concentration is sufficient for the reliable analysis using $40 \mu \mathrm{M}$ DCPIP mediator

all the samples, while the decrease in the excitation energy frequency to $0.05 \mathrm{~Hz}$ (experiment 9) allowed reaching the saturation level of the $-\Delta A_{0}$ signal in all the samples. Evidently, the combination of $4-10 \mathrm{mM}$ ascorbate with $4 \mu \mathrm{M}$ DCPIP mediator was enough to recover fully the neutral $P$ state at $0.05 \mathrm{~Hz}$ excitation frequency for all the samples.

Further increase in the DCPIP concentration to $40 \mu \mathrm{M}$ led to the observation that even with the $0.5-\mathrm{Hz}$ repetition rate, maximal $-\Delta A_{0}$ signals were reached for $C$. merolae and $C$. reinhardtii PSI samples (experiment 10 ). Under these conditions, a further increase in the excitation energy (experiment 11) or decrease in the excitation frequency (experiment 12) did not improve the signal in any sample analyzed. We conclude that the combination of 4-10 $\mathrm{mM}$ ascorbate with $40 \mu \mathrm{M}$ DCPIP at $0.5-\mathrm{Hz}$ and $1-\mathrm{mJ}$ excitations yielded the most optimal conditions for all the samples under study allowing to obtain the highest possible (saturated) $P^{+}$signal. The only exception was the cyanobacterial SM sample for which the $P^{+}$signals were smaller in the presence of $40 \mu \mathrm{M}$ DCPIP compared to the $4 \mu \mathrm{M}$ DCPIP (Fig. 6A) due to unknown reasons.

The highest obtained (saturated) initial $P^{+}$signal was 5-6 mOD for the cyanobacterial and red algal CmH PSI sample, $\sim 9 \mathrm{mOD}$ for the red algal $\mathrm{CmM}$ and $16 \mathrm{mOD}$ for green algal Cr PSI samples (Fig. 6). These values are roughly proportional to the fraction of primary donors per number of antenna chlorophylls $\left(P / N_{\text {Chls }} ;\right.$ Fig. 7$)$ in the individual samples and maximal expected ones. Despite the identical optical density of all the samples at the $O_{y}$ band maximum $\left(\mathrm{OD}_{\sim 680 \mathrm{~nm}, 1 \mathrm{~cm}}=1\right)$, the strict proportionality of these quantities was not expected since the differential molar extinction coefficient of the primary donor at $\sim 700 \mathrm{~nm}\left(\Delta \varepsilon_{700 \mathrm{~nm}}\left(P^{+}-P\right)\right)$

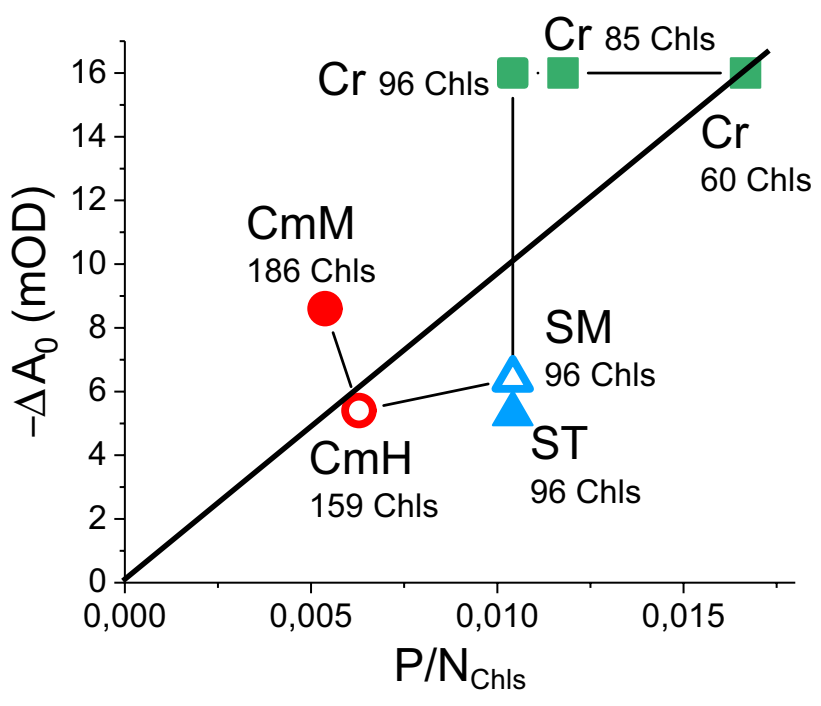

Fig. 7 Dependence of the maximal initial amplitude $\left(-\Delta A_{0}\right)$ for each of the PSI preparation on the $P / N_{\mathrm{Chls}}$ ratio in each type of PSI complex (estimated from the kinetics measured in 54-130-ms time windows). $N_{\text {Chls }}$ is the number of antenna Chls per one primary donor for the individual type of PSI whereas $P / N_{\text {Chls }}$ is its reciprocal; the $N_{\text {Chls }}$ number is shown at each label identifying the individual type of PSI; the three different values of $N_{\text {Chls }}$ for the Cr PSI come from three different references (96 Chls - [43], 85 Chls - [42], 60 Chls - [41]). The thick straight line is a theoretical dependence obtained under the assumption that the $\Delta A_{700 \mathrm{~nm}}\left(P^{+}-P\right)=\Delta A_{\sim 680 \mathrm{~nm}}\left(\mathrm{Chl}^{*-\mathrm{Chl}}\right)$ and that excitation pulse was saturating (oxidizing primary donors in all probed PSI complexes across the cuvette)

relative to the molar extinction coefficient of the antenna Chls at the $Q_{y}$ band maximum may be different for selected species and preparations.

Recently, it has been shown that in contrast to the PSI samples of the present study, even a low $(2 \mathrm{mM})$ concentration of ascorbate alone (without any additional mediators) was sufficient to observe the maximal $\Delta A$ signal from $P^{+}$ in the case of PSI complex from a desert green microalga Chlorella ohadii grown under high light intensity conditions [54]. On the other hand, PSI particles isolated from the same species but grown under low light conditions required the addition of mediators to show the maximal $\Delta A$ signal from $P^{+}$. The same study showed that the two types of illumination conditions affected the structure, pigment composition, and electron transfer in the respective RCs. This documents the importance of the growth conditions, especially illumination intensity, which may influence not only the remodeling of the PSI structure (e.g., [38]), the intrinsic energy transfer pathways (e.g., [39]), but also the accessibility of the exogenous electron carriers to the PSI ETC species, as the present study clearly demonstrates.

3.2.2.2 Average time constant of charge recombination The second column of panels in Fig. 6 (B, F, J, N, R) 
compares the average time constants of charge separation calculated from Eq. 3. For the cyanobacterial samples, these constants range from $\sim 1.5 \mathrm{~ms}(\mathrm{SM})$ to $\sim 2 \mathrm{~ms}(\mathrm{ST})$ and do not show any clear dependence on the experimental conditions. They are assigned to $P^{+} F_{X}{ }^{-} \rightarrow P F_{X}$ recombination. The extraordinarily big values of $\tau_{\text {av }}$ for experiments number 3 and 7 for the ST sample (Fig. 6F) are artifacts resulting from a limited signal-to-noise ratio of the data. Indeed, the presence of these particularly slow phases is correlated with the lack of non-decaying components in the respective fits (Fig. 6G; not resolved non-decaying components artificially increase $\tau_{\text {av }}$ ).

For the remaining samples $(\mathrm{CmH}, \mathrm{CmM}$ and $\mathrm{Cr}-$ Fig. $6 \mathrm{~J}$, $\mathrm{N}, \mathrm{R}$ ), the average time constant shows a gradual increase upon the reduction power of the solution (from experiment 1 to experiment 12). This effect is assigned to the heterogeneity of PSI complexes discussed above. The small fractions of PSI complexes yielding the $P^{+}$signal even in the absence of redox mediators (experiments 1-3) contain the significant amount of most likely structurally perturbed proteins with charge separation limited to the state $P^{+} F_{X}{ }^{-}$followed by the fast 1-2 $\mathrm{ms}^{+} F_{X}{ }^{-} \rightarrow P F_{X}$ charge recombination (compare Figs. $4 \mathrm{C}, \mathrm{D}$ ), which accelerates the average recombination values (in the kinetics recorded without mediators, 1-3). This effect is preserved in all the following experiments (4-12), but its relative contribution decreases systematically with increasing the reduction power. The two extraordinarily large values of $\tau_{\mathrm{av}}$ for experiments number 1 and 4 for the Cr sample (Fig. 6R) are artifacts, similar to those shown in Fig. $6 \mathrm{~F}$ due to the limited signal-to-noise ratio and unresolved offset (Fig. 6S).

The average $P^{+}$decay time, $\tau_{\text {av }}$, at high reduction power of solution (experiments 10-12), approaches the values of $40 \mathrm{~ms}$ for $\mathrm{CmH}$ and CmM PSI and $80 \mathrm{~ms}$ for Cr PSI samples. Both these values can be confidently assigned to the $P^{+}\left(F_{A} / F_{B}\right)^{-} \rightarrow P\left(F_{A} / F_{B}\right)$ charge recombination process [36, 50].

\subsubsection{Non-decaying component and the PSI electron} escape time constant As noted above, the non-decaying component (offset, the third column of panels in Fig. 6) was for some kinetics data difficult to resolve. However, for the majority of collected kinetic traces, it was resolved. In the case of cyanobacterial samples, the offset was generally smaller for the fraction of PSI complexes yielding the $P^{+}$ signal even without mediators (experiments 1-3) compared to the offset resolved when the mediators were added (experiments 4-12). This is because in the fraction without mediators, charge recombination is much faster than the electron escape from $F_{X}{ }^{-}$outside PSI (see above). Thus, either the offset is zero (charge recombination fully recovers neutral $P$ on the time scale of the measured kinetics) or it is non-zero but then it is assigned to particularly slow charge recombi- nation (as argued above). For the samples with mediators, the offset is assigned to ET from PSI to exogenous acceptor with a contribution of ET from the electrolyte to $P^{+}$, as discussed above, particularly at the highest reduction power of the electrolyte. For the $C$. merolae and $C$. reinhardtii PSI preparations (Fig. 6K, O, S) the assignments are similar as in the case of the cyanobacterial samples: in samples with added mediators, the offset may originate from ET from PSI to the external mediator (and from mediator to PSI), whereas in the samples without mediators - most likely it originates from the slow charge recombination. Regarding the experiments with mediators (experiments 4-12), there is a tendency, for $\mathrm{Cm}$ and $\mathrm{Cr}$ samples, of a decrease in the offset with increasing reduction power (Fig. 6K, O, S). This effect seems to reveal the dependence of the rate of ET from the electrolyte to $P^{+}, k_{P}$, on the reduction power: at the high reduction power this ET occurs on a time scale comparable with the time window of the experiment.

The data presented in the last column in Fig. 6, time constants of electron escape from PSI $\left(\tau_{\text {out }}\right)$ estimated from Eq. 2, are correlated with the values of the offset for the respective samples because these two parameters are inversely proportional to each other (Eq. 2). For the cyanobacterial samples, SM and ST, this parameter oscillates around $50 \mathrm{~ms}$ for most of the experimental conditions. For the $\mathrm{Cm}$ and $\mathrm{Cr}$ samples, the electron escape rate decelerates with increasing the reduction power of the solvent: for Cm samples, $\tau_{\text {out }}$ increases from $\sim 500 \mathrm{~ms}$ to single seconds, whereas for Cr PSI, $\tau_{\text {out }}$ it is generally shorter and increases from $\sim 50$ to $\sim 200 \mathrm{~ms}$ (Fig. 6L, P, T). As discussed above for the offset, the reduction power-dependence of the $\tau_{\text {out }}$ parameter defined in Eq. 2 is due to the variable rate of ET from the electrolyte to $P^{+}$. It should be noted that the time constant $\tau_{\text {out }}$ was estimated only for experiments 4-12. This is consistent with the assumption justified above that in the experiments without mediators (1-3) electrons cannot escape from the PSI fraction observable in the transient absorption experiments.

\subsubsection{Contribution of $P^{+} A_{1}{ }^{-} \rightarrow P A_{1}$ charge recombination to $P^{+}$decay kinetics}

As shown in Table 1, the shortest kinetic component resolved for the SM and ST samples is $\sim 1 \mathrm{~ms}$, which is characteristic of the $P^{+} F_{X}{ }^{-} \rightarrow P F_{X}$ charge recombination. To check whether there is any $P^{+} A_{1}{ }^{-} \rightarrow P A_{1}$ charge recombination in these complexes, we performed transient absorption measurements with the higher temporal resolution by decreasing the size of the temporal window to $0.5 \mathrm{~ms}$. In these experiments (with saturating concentrations of external mediators), we were able to resolve an additional decay phase of 10-30 $\mu$ s assigned to the $P^{+} A_{1}{ }^{-} \rightarrow P A_{1}$ charge recombination. Its contribution to the total decay was only $10 \%$ for 
the SM and $16 \%$ for the ST samples (data not shown). This additional decay channel is consistent with the relatively low initial $\Delta A$ signals recorded with a lower temporal resolution (Fig. 7).

Transient absorption experiments performed in a 0.5 ms time window with more intact PSI complexes from $C$. merolae demonstrated a low contribution of $P^{+} A_{1}{ }^{-} \rightarrow P A_{1}$ charge recombination $(\sim 50 \mu \mathrm{s})$ of $2-4 \%$ of the entire PSI population from this species (data not shown). Thus, for the C. merolae and similarly for $C$. reinhardtii PSI complexes, we estimate that the full charge separation, i.e., formation of the $P^{+}\left(F_{A} / F_{B}\right)^{-}$species occurs in $\sim 90 \%$ of the PSI particle population. This number is comparable with the efficiency of the "truncated" charge separation $\left(P^{+} F_{X}^{-}\right)$in cyanobacterial PSI (84-90\%).

\section{Conclusion}

Our results show that the cyanobacterial PSI complexes stored at $-20{ }^{\circ} \mathrm{C}$ without glycerol are devoid of the functionally coupled PsaC subunit coordinating the $F_{A}$ and $F_{B}$ terminal electron acceptors. Whether or not such preparations contain a physically attached $\mathrm{PsaC}$ subunit remains to be clarified. The final electron acceptor in these complexes seems to be $F_{X}$. This is demonstrated by the lack of charge recombination phases longer than few milliseconds (except for a small 5\% fraction in the ST sample). Despite the negative effects on the structural integrity of cyanobacterial PSI upon storage at low temperature without glycerol (cryoprotectant), such protein complexes may still be useful for photovoltaic applications since ET from $F_{X}{ }^{-}$outside PSI is faster $(\sim 50 \mathrm{~ms})$ compared with ET from $\left(F_{A} / F_{B}\right)^{-}$outside PSI for the more intact PSI preparations from $C$. merolae and $C$. reinhardtii ( $>200 \mathrm{~ms}$ ). An additional advantage of using the disturbed form of PSI in photovoltaics is the lower redox potential of $F_{X}{ }^{-}$compared to $\left(F_{A} / F_{B}\right)^{-}$. A similar rationale was successfully applied by oriented molecular wiring of PSI to the ITO surface through an even more reducing site, i.e., by replacing the native phylloquinone cofactor with the vitamin $\mathrm{K} 1$ analog, a naphthoquinone moiety [55].

The $90 \%$ efficiencies of full charge separation in all the samples in solution should be compared with the estimated IQE values measured for the electrodes functionalized with the same PSI particles, ranging from $\sim 0.3$ to $\sim 0.5 \%$ (Fig. 3). In the first approximation, we assumed that results obtained in solution (Table 1) are conserved after deposition of PSI on electrodes. Under such an assumption, the critical factor is the offset parameter (const in Eq. 1) which may be interpreted as the upper limit of electron escape probability from PSI to the electrolyte in the PEC device. Thus, this parameter shows the first bottleneck for obtaining the high photocurrent output in the PSI-based PEC systems.
The offset parameter equals on average $~ 4 \%$ for both cyanobacterial PSI and PSI from C. merolae and 30-40\% for $C$. reinhardtii (Tab. 1, Fig. 6). Thus, in the case of Synechocystis and C. merolae, the dominating factor limiting the photocurrent generation is the internal charge recombination (compare $4 \%$ efficiency of electron transfer outside PSI with $\sim 90 \%$ efficiency of complete charge separation). However, even $4 \%$ efficiency is still $\sim 10$ times higher than $\sim 0.3-0.5 \%$ IQE measured for the electrodes functionalized with these samples. Thus, it can be stipulated that most of the photo-generated electrons transferred outside the cyanobacterial and red algal PSI complexes (90\%) do not reach the counter electrode of PEC but instead may undergo electric short-circuiting with PSI particles present in the photoactive layer. The situation seems to be somewhat different for the $C$. reinhardtii PSI, for which efficiency of electron transfer outside PSI is as much as $30-40 \%$, which means that every second, every third electron generated in PSI is transferred outside to the electrolyte. On the other hand, the chance that the electron that is transferred outside PSI will reach the counter electrode is only $\sim 1 \%$ (compare $30-40 \%$ offset with $0.4 \%$ IQE), most likely, as above, due to electric short-circuiting between the adjacent Cr PSI particles immobilized on the electrode surface. It would be therefore important to conduct similar transient absorption studies in a thin film of PSI complexes immobilized on FTO for comparison with the ET kinetics obtained for the PSI samples in solution. Although this is an extremely demanding experiment, we believe it is doable and this type of analysis would provide valuable insight into the ET limiting processes occurring within the PSI-based photo-electrodes and the bio-PEC devices.

Our studies indicate that to increase the IQE of PSI-based PECs, the two main bottlenecks should be overcome. First, the efficiency of electron uptake from PSI by electrolyte should be increased, particularly for the PSI biophotocatalysts from Synechocystis and C. merolae. Second, the probability of electric short-circuiting of electrons transferred from PSI to electrolyte should be limited. The latter limitation can be overcome through nano-structuring of the PSI complexes within thin films, e.g., through the use of DNA origami [56], the orienting biotic conductive thin layers (made of, e.g., cytochrome $c$; [14, 57-59]) or organic molecular wires $[60,61]$.

Acknowledgements Part of this work was supported by funding from the National Science Centre, Poland (OPUS14 Grant No. UMO2017/27/B/ST5/00472 to J.K.).

\section{Declarations}

Conflict of interest On behalf of all authors, the corresponding author states that there is no conflict of interest. 
Open Access This article is licensed under a Creative Commons Attribution 4.0 International License, which permits use, sharing, adaptation, distribution and reproduction in any medium or format, as long as you give appropriate credit to the original author(s) and the source, provide a link to the Creative Commons licence, and indicate if changes were made. The images or other third party material in this article are included in the article's Creative Commons licence, unless indicated otherwise in a credit line to the material. If material is not included in the article's Creative Commons licence and your intended use is not permitted by statutory regulation or exceeds the permitted use, you will need to obtain permission directly from the copyright holder. To view a copy of this licence, visit http://creativecommons.org/licenses/by/4.0/.

\section{References}

1. Nguyen, K., \& Bruce, B. D. (2014). Growing green electricity: Progress and strategies for use of Photosystem I for sustainable photovoltaic energy conversion. Biochimica et Biophysica Acta (BBA) - Bioenergetics, 9(1837), 1553-1566. https://doi.org/10. 1016/j.bbabio.2013.12.013

2. Ravi, S. K., \& Tan, S. C. (2015). Progress and perspectives in exploiting photosynthetic biomolecules for solar energy harnessing. Energy and Environmental Science, 8, 2551-2573. https:// doi.org/10.1039/C5EE01361E

3. Friebe, V. M., \& Frese, R. N. (2017). Photosynthetic reaction center-based biophotovoltaics. Current Opinion in Electrochemistry, 5(1), 126-134. https://doi.org/10.1016/j.coelec.2017.08.001

4. Musazade, E., Voloshin, R., Brady, N., Mondal, J., Atashova, S., Zharmukhamedov, D. K., Huseynova, I., Ramakrishna, S., Najafpour, M. M., Shen, J. R., Bruce, B. D., \& Allakhverdiev, S. I. (2018). Biohybrid solar cells: Fundamentals, progress, and challenges. Journal of Photochemistry and Photobiology C: Photochemistry Reviews, 35, 134-156. https://doi.org/10.1016/j.jphot ochemrev.2018.04.001

5. Wolfe, K. D., Dervishogullari, D., Passantino, J. M., Stachurski, C. D., Jennings, G. K., \& Cliffel, D. E. (2020). Improving the stability of photosystem I-based bioelectrodes for solar energy conversion. Current Opinion in Electrochemistry, 19, 27-34. https://doi.org/10.1016/j.coelec.2019.09.009

6. Teodor, A. H., \& Bruce, B. D. (2020). Putting Photosystem I to work: Truly green energy. Trends in Biotechnology, 38(12), 1329-1342. https://doi.org/10.1016/j.tibtech.2020.04.004

7. Blankenship, R. E. (2021). Molecular mechanisms of photosynthesis. Kluwer Academic Publishers.

8. Nelson, N., \& Junge, W. (2015). Structure and energy transfer in photosystems of oxygenic photosynthesis. Annual Review of Biochemistry, 84, 659-683. https://doi.org/10.1146/annurev-bioch em-092914-041942

9. Ciesielski, P. N., Hijazi, F. M., Scott, A. M., Faulkner, C. J., Beard, L., Emmet, K., Rosenthal, S. J., Cliffel, D., \& Jennings, G. K. (2010). Photosystem I-based biohybrid photoelectrochemical cells. Bioresource Technology, 101, 3047-3053. https://doi.org/ 10.1016/j.biortech.2009.12.045

10. Mershin, A., Matsumoto, K., Kaiser, L., Yu, D., Vaughn, M., Nazeeruddin, M. K., Bruce, B. D., Graetzel, M., \& Zhang, S. (2012). Self-assembled Photosystem-I biophotovoltaics on nanostructured $\mathrm{TiO}_{2}$ and $\mathrm{ZnO}$. Scientific Reports, 2, 234. https://doi. org/10.1038/srep00234

11. Ocakoglu, K., Krupnik, T., van den Bosch, B., Harputlu, E., Gullo, M. P., Olmos, J. D. J., Yildirimcan, S., Gupta, R. K., Yakuphanoglu, F., Barbieri, A., Reek, J. N. H., \& Kargul, J. (2015). Photosystem I-based biophotovoltaics on nanostructured hematite.
Advanced Functional Materials, 25(9), 1337-1337. https://doi. org/10.1002/adfm.201401399

12. Yu, D., Wang, M., Zhu, G., Ge, B., Liu, S., \& Huang, F. (2015). Enhanced photocurrent production by bio-dyes of photosynthetic macromolecules on designed $\mathrm{TiO}_{2}$ film. Scientific Reports, 5 , 9375. https://doi.org/10.1038/srep09375

13. Gizzie, E. A., LeBlanc, G., Jennings, G. K., \& Cliffel, D. E. (2015). Electrochemical preparation of Photosystem I-polyaniline composite films for biohybrid solar energy conversion. ACS Applied Materials and Interfaces, 7(18), 9328-9335. https://doi. org/10.1021/acsami.5b01065

14. Stieger, K. R., Ciornii, D., Kolsch, A., Hejazi, M., Lokstein, H., Feifel, S. C., Zouni, A., \& Lisdat, F. (2016). Engineering of supramolecular photoactive protein architectures: The defined coassembly of photosystem I and cytochrome $c$ using a nanoscaled DNA-matrix. Nanoscale, 8, 10695-10705. https://doi.org/10. 1039/C6NR00097E

15. Stieger, K. R., Feifel, S. C., Lokstein, H., Hejazi, M., Zouni, A., \& Lisdat, F. (2016). Biohybrid architectures for efficient light-tocurrent conversion based on photosystem I within scalable 3D mesoporous electrodes. Journal of Materials Chemistry A, 43, 17009-17017. https://doi.org/10.1039/C6TA07141D

16. Szewczyk, S., Białek, R., Burdziński, G., \& Gibasiewicz, K. (2020). Photovoltaic activity of electrodes based on intact photosystem I electrodeposited on bare conducting glass. Photosynthesis Research, 144, 1-12. https://doi.org/10.1007/ s11120-020-00722-1

17. Ciesielski, P. N., Scott, A. M., Faulkner, C. J., Berron, B. J., Cliffel, D. E., \& Jennings, G. K. (2008). Functionalized nanoporous gold leaf electrode films for the immobilization of photosystem I. ACS Nano, 2(12), 2465-2472. https://doi.org/10.1021/nn800389k

18. Ciesielski, P. N., Faulkner, C. J., Irwin, M. T., Gregory, J. M., Tolk, N. H., Cliffel, D. E., \& Jennings, G. K. (2010). Enhanced photocurrent production by Photosystem I multilayer assemblies. Advanced Functional Materials, 20, 4048-4054. https://doi.org/ 10.1002/adfm.201001193

19. Gunther, D., LeBlanc, G., Prasai, D., Zhang, J. R., Cliffel, D. E., Bolotin, K. L., \& Jennings, G. K. (2013). Photosystem I on graphene as a highly transparent, photoactive electrode. Langmuir, 29, 4177-4180. https://doi.org/10.1021/la305020c

20. Feifel, S. C., Stieger, K. R., Lokstein, H., Lux, H., \& Lisdat, F. (2015). High photocurrent generation by Photosystem I on artificial interfaces composed of $\pi$-system-modified graphene. Journal of Material Chemistry A, 3, 12188-12196. https://doi.org/10. 1039/C5TA00656B

21. Shah, V. B., Henson, W. R., Chadha, T. S., Lakin, G., Liu, H., Blankenship, R. E., \& Biswas, P. (2015). Linker-free deposition and adhesion of Photosystem I onto nanostructured $\mathrm{TiO}_{2}$ for biohybrid photoelectrochemical cells. Langmuir, 31(5), 1675-1682. https://doi.org/10.1021/la503776b

22. Wang, P., Frank, A., Zhao, F., Szczesny, J., Junqueira, J. R. C., Zacarias, S., Ruff, A., Nowaczyk, M. M., Pereira, I. A. C., R o gner, M., Conzuelo, F., \& Schuhmann, W. (2020). Closing the gap for electronic short-circuiting: Photosystem I mixed monolayers enable improved anisotropic electron flow in biophotovoltaic devices. Angewandte Chemie International Edition, 60, 20002006. https://doi.org/10.1002/anie.202008958

23. Zhao, F., Wang, P., Ruff, A., Hartmann, V., Zacarias, S., Pereira, I. A. C., Nowaczyk, M. M., Rögner, M., Conzuelo, F., \& Schuhmann, W. (2019). A Photosystem I monolayer with anisotropic electron flow enables Z-scheme like photosynthetic water splitting. Energy and Environmental Science, 12, 3133-3143. https:// doi.org/10.1039/C9EE01901D

24. Jordan, P., Fromme, P., Witt, H. T., Klukas, O., Saenger, W., \& Krauss, N. (2001). Three-dimensional structure of cyanobacterial 
Photosystem I at 2.5A resolution. Nature, 411, 909-917. https:// doi.org/10.1021/ja0587749

25. Amunts, A., Drory, O., \& Nelson, N. (2007). The structure of a plant Photosystem I supercomplex at 3.4 A resolution. Nature, 447, 58-63. https://doi.org/10.1038/nature05687

26. Qin, X., Suga, M., Kuang, T., \& Shen, J. R. (2015). Structural basis for energy transfer pathways in the plant PSI-LHCI supercomplex. Science, 348, 989-995. https://doi.org/10.1126/science. aab0214

27. Qin, X., Pi, X., Wang, W., Han, G., Zhy, L., Liu, M., Cheng, L., Shen, J. R., Kuang, T., \& Sui, S. F. (2019). Structure of a green algal Photosystem I in complex with a large number of light-harvesting complex I subunits. Nature Plants, 5, 263-272. https://doi. org/10.1038/s41477-019-0379-y

28. Makita, H., \& Hastings, G. (2017). Inverted-region electron transfer as a mechanism for enhancing photosynthetic solar energy conversion efficiency. Proceedings of National Academy of Sciences of the United States of America, 35(114), 9267-9272. https://doi. org/10.1073/pnas.1704855114

29. Brettel, K. (1997). Electron transfer and arrangement of the redox cofactors in photosystem I. Biochimica et Biophysica Acta (BBA) - Bioenergetics, 1318, 322-373.

30. Brettel, K., \& Leibl, W. (2001). Electron transfer in Photosystem I. Biochimica et Biophysica Acta (BBA) - Bioenergetics, 1507, 100-114. https://doi.org/10.1016/S0005-2728(01)00202-X

31. Savikhin, S. (2006). In J. H. Golbeck (Ed.), Photosystem I: The light-driven plastocyanin: Ferredoxin oxidoreductase (155175). Springer.

32. Guergova-Kuras, M. G., Boudreaux, B., Joliot, A., Joliot, P., $\&$ Redding, K. (2001). Evidence for two active branches for electron transfer in photosystem I. Proceedings of National Academy of Sciences of the United States of America, 8(98), 4437-4442. https://doi.org/10.1073/pnas.081078898

33. Ramesh, V. M., Gibasiewicz, K., Lin, S., Bingham, S. E., \& Webber, A. N. (2004). Bidirectional electron transfer in Photosystem I: Accumulation of $A_{0^{-}}$in $A$-side or B-side mutants of the axial ligand to chlorophyll $A_{0}$. Biochemistry, 43(5), 13691375. https://doi.org/10.1021/bi0354177

34. Dashdorj, N., Xu, W., Cohen, R. O., Golbeck, J. H., \& Savikhin, S. (2005). Asymmetric electron transfer in cyanobacterial Photosystem I: Charge separation and secondary electron transfer dynamics of mutations near the primary electron acceptor $A_{0}$. Biophysical Journal, 88(2), 1238-1249. https://doi.org/10.1529/ biophysj.104.050963 Epub 2004 Nov 12.

35. Santabarbara, S., Kuprov, I., Poluektov, O., Casal, A., Russell, C. A., Purton, S., \& Evans, M. C. (2010). Directionality of electron-transfer reactions in Photosystem I of prokaryotes: Universality of the bidirectional electron-transfer model. Journal of Physical Chemistry B, 114(46), 15158-15171. https://doi.org/ 10.1021/jp1044018 Epub 2010 Oct 26 PMID: 20977227.

36. Kurashov, V., Gorka, M., Milanovsky, G. E., Johnson, T. W., Cherepanov, D. A., Semenov, A. Y., \& Golbeck, J. H. (2018). Critical evaluation of electron transfer kinetics in P700-FA/ FB, P700-FX, and P700-A1 Photosystem I core complexes in liquid and in trehalose glass. Biochimica et Biophysica Acta (BBA) - Bioenergetics, 1859, 1288-1301. https://doi.org/10. 1016/j.bbabio.2018.09.367

37. Szewczyk, S., Giera, W., D'Haene, S., van Grondelle, R., \& Gibasiewicz, K. (2017). Comparison of excitation energy transfer in cyanobacterial Photosystem I in solution and immobilized on conducting glass. Photosynthesis Research, 132(2), 111-126. https://doi.org/10.1007/s11120-016-0312-4

38. Haniewicz, P., Abram, M., Nosek, L., Kirkpatrick, J., ElMohsnawy, E., Olmos, J. D. J., Kouřil, R., \& Kargul, J. M. (2018). Molecular mechanisms of photoadaptation of Photosystem I supercomplex from an evolutionary cyanobacterial/ algal intermediate. Plant Physiology, 176, 1433-1451. https:// doi.org/10.1104/pp.17.01022

39. Abram, M., Białek, R., Szewczyk, S., Karolczak, J., Gibasiewicz, K., \& Kargul, J. (2020). Remodeling of excitation energy transfer in extremophilic red algal PSI-LHCI complex during light adaptation. Biochimica et Biophysica Acta (BBA) - Bioenergetics, 1(1861), 148093. https://doi.org/10.1016/j.bbabio. 2019.148093

40. Giera, W., Szewczyk, S., McConnell, M. D., Snellenburg, J., Redding, K. E., van Grondelle, R., \& Gibasiewicz, K. (2014). Excitation dynamics in Photosystem I from Chlamydomonas reinhardtii. Comparative studies of isolated complexes and whole cells. Biochimica et Biophysica Acta (BBA) - Bioenergetics, 1837, 1756-1768. https://doi.org/10.1016/j.bbabio.2014.06.004

41. Owens, T. G., Webb, S. P., Mets, L., Alberte, R. S., \& Fleming, G. R. (1989). Antenna structure and excitation dynamics in photosystem I. Studies with mutants of Chlamydomonas reinhardtii lacking photosystem II. Biophysical Journal, 56, 95-106. https:// doi.org/10.1016/S0006-3495(89)82654-2

42. Gibasiewicz, K., Ramesh, V. M., Melkozernov, A. N., Lin, S., Woodbury, N. W., Blankenship, R. E., \& Webber, A. N. (2001). Excitation dynamics in the core antenna of PS I from Chlamydomonas reinhardtii CC 2696 at room temperature. Journal of Physical Chemistry B, 105, 11498-11506. https://doi.org/10. 1021/jp012089g

43. Su, X., Ma, J., Pan, X., Zhao, X., Chang, W., Liu, Z., Zhang, X., $\&$ Li, M. (2019). Antenna arrangement and energy transfer pathways of a green algal photosystem-I-LHCI supercomplex. Nature Plants, 5, 273-281. https://doi.org/10.1038/s41477-019-0380-5

44. Burdzinski, G., Bayda, M., Hug, G. L., Majchrzak, M., Marciniec, B., \& Marciniak, B. (2011). Time-resolved studies on the photoisomerization of a phenylene-silylene- vinylene type compound in its first singlet excited state. Journal of Luminescence, 131, 577-580. https://doi.org/10.1016/j.jlumin.2010.10.031

45. Sapper, H., Kang, S. O., Paul, H. H., \& Lohmann, W. (1982). The reversibility of the vitamin $\mathrm{C}$ redox system: Electrochemical reasons and biological aspects. Z. Naturforsch C Bioscience, 37(10), 942-946. https://doi.org/10.1515/znc-1982-1015

46. Izawa, S. (1980). Acceptors and donors and chloroplast electron transport. Methods in Enzymology, 69, 413-434. https://doi.org/ 10.1016/S0076-6879(80)69041-7

47. Kennet, E. C., \& Kuchel, P. W. (2003). Redox reactions and electron transfer across the red cell membrane. International Union of Biochemistry and Molecular Biology, 55(7), 375-385. https:// doi.org/10.1080/15216540310001592843

48. Efimov, I., Parkin, G., Millett, E. S., Glenday, J., Chan, C. K., Weedon, H., Randhawa, H., Basran, J., \& Raven, E. L. (2014). A simple method for the determination of reduction potentials in heme proteins. FEBS Letters, 588(5), 701-704. https://doi.org/10. 1016/j.febslet.2013.12.030

49. Nakamura, A., Suzawa, T., \& Watanabe, T. (2004). Spectroelectrochemical determination of the redox potential of P700 in spinach with an optically transparent thin-layer electrode. Chemistry Letters, 33(6), 688-689. https://doi.org/10.1246/cl.2004.688

50. Vassiliev, I. R., Jung, Y. S., Mamedov, M. D., Semenov, A. Y., \& Golbeck, J. H. (1997). Near-IR absorbance changes and electrogenic reactions in the microsecond-to-second time domain in photosystem I. Biophysical Journal, 72, 301-315. https://doi.org/ 10.1016/S0006-3495(97)78669-7

51. Passantino, J. M., Wolfe, K. D., Simon, K. T., Cliffel, D. E., \& Jennings, G. K. (2020). Photosystem I enhances the efficiency of a natural, gel-based dye-sensitized solar cell. ACS Applied Bio Materials, 3(7), 4465-4473. https://doi.org/10.1021/acsabm. $0 \mathrm{c} 00446$

52. Trubitsin, B. V., Mamedov, M. D., Semenov, Y. A., \& Tikhonov, A. N. (2014). Interaction of ascorbate with Photosystem 
I. Photosynthesis Research, 122(2), 215-231. https://doi.org/10. 1007/s11120-014-0023-7

53. Gourovskaya, K. N., Mamedov, M. D., Vassiliev, I. R., Golbeck, J. H., \& Semenov, A. Y. (1997). Electrogenic reduction of the primary electron donor $\mathrm{P} 700^{+}$in Photosystem I by redox dyes. FEBS Letters, 414(2), 193-196. https://doi.org/10.1016/s0014-5793(97) 00994-0

54. Caspy, I., Neumann, E., Fadeeva, M., Liveanu, V., Savitsky, A., Frank, A., Kalisman, Y. L., Shkolnisky, Y., Murik, O., Treves, H., Hartmann, V., Nowaczyk, M. M., Schuhmann, W., Rogner, M., Willner, I., Kaplan, A., Schuster, G., Nelson, N., Lubitz, W., \& Nechushtai, R. (2021). Cryo-EM photosystem I structure reveals adaptation mechanisms to extreme high light in Chlorella ohadii. Nature Plants, 7, 1314-1322. https://doi.org/10.1038/ s41477-021-00983-1

55. Miyachi, M., Yoshinori, Y., Tetsu, Y., Hiroshi, N., Michinao, I., Masae, K., Masako, I., \& Yasunori, I. (2009). Surface immobilization of PSI using vitamin K1-like molecular wires for fabrication of a bio-photoelectrode. Journal of Nanoscience and Nanotechnology, 9(3), 1722-1726. https://doi.org/10.1166/jnn.2009.SI06

56. Kamińska, I., Bohlen, J., Yaadav, R., Schüler, P., Raab, M., Schröder, T., Zähringer, J., Zielonka, K., Krause, S., \& Tinnefeld, P. (2021). Graphene energy transfer for single-molecule biophysics, biosensing, and super-resolution microscopy. Advanced Materials, 33, 2101099. https://doi.org/10.1002/adma.202101099

57. Kiliszek, M., Harputlu, E., Szalkowski, M., Kowalska, D., Ünlü, C. G., Haniewicz, P., Abram, M., Wiwatowski, K., NiedziółkaJönsson, J., Maćkowski, S., Ocakoglu, K., \& Kargul, J. M. (2018).
Orientation of photosystem I on graphene through cytochrome c553 leads to improvement in photocurrent generation. Journal of Materials Chemistry, 6, 18615-18626. https://doi.org/10.1039/ C8TA02420K

58. Szalkowski, M., Harputlu, E., Kiliszek, M., Unlu, C. G., Maćkowski, S., Ocakoglu, K., Kargul, J., \& Kowalska, D. (2020). Plasmonic enhancement of photocurrent generation in a Photosystem I-based hybrid electrode. Journal of Materials Chemistry C, 8, 5807. https://doi.org/10.1039/C9TC06983F

59. Izzo, M., Jacquet, M., Fujiwara, T., Harputlu, E., Mazur, R., Wróbel, P., Góral, T., Unlu, C. G., Ocakoglu, K., Miyagishima, S., \& Kargul, J. (2021). Development of a Novel Nanoarchitecture of the Robust Photosystem I from a Volcanic Microalga Cyanidioschyzon merolae on Single Layer Graphene for Improved Photocurrent Generation. International Journal of Molecular Sciences, 22, 8396. https://www.mdpi.com/1422-0067/22/16/8396/htm

60. Lubner, C. E., Grimme, R., Bryant, D. A., \& Golbeck, J. H. (2009). Wiring Photosystem I for direct solar hydrogen production. Biochemistry, 49, 404-414. https://doi.org/10.1021/bi901 $704 \mathrm{v}$

61. Jacquet, M., Izzo, M., Osella, S., Kozdra, S., Michałowski, P. P., Gołowicz, D., Kazimierczuk, K., Gorzkowski, M. T., Lewera, A., Teodorczyk, M., Trzaskowski, B., Jurczakowski, R., Gryko, D. T., \& Kargul, J. (2021). Development of a universal conductive platform for anchoring photo- and electroactive proteins using organometallic terpyridine molecular wires. Nanoscale, 13, 9773. https://doi.org/10.1039/D0NR08870F

\section{Authors and Affiliations}

\section{Alice Goyal ${ }^{1}$ - Sebastian Szewczyk ${ }^{1}$. Gotard Burdziński ${ }^{1}$ - Mateusz Abram ${ }^{2,3}$. Joanna Kargul ${ }^{2}$. Krzysztof Gibasiewicz ${ }^{1}$ (D)}

1 Faculty of Physics, Adam Mickiewicz University in Poznań, ul. Uniwersytetu Poznańskiego 2, 61-614 Poznań, Poland

2 Solar Fuels Laboratory, Centre of New Technologies, University of Warsaw, Banacha 2C, 02-097 Warsaw, Poland
3 Faculty of Biology, University of Warsaw, Miecznikowa 1, 02-096 Warsaw, Poland 